\title{
Origin and K-Ar age of the phreatomagmatic breccia at the Trepča Pb-Zn-Ag skarn deposit, Kosovo: Implications for ore-forming processes
}

\author{
Sabina Strmić Palinkaš ${ }^{1}{ }^{*}$, Ladislav Palinkaš², Magda Mandić ${ }^{3}$, Zvjezdana Roller-Lutz ${ }^{4}$, Zoltan Pécskay5, Gani \\ Maliqi ${ }^{6}$ and Vladimir Bermanec ${ }^{2}$
}

\author{
${ }^{1}$ University of Tromsø, Faculty of Science and Technology, Department of Geology, Dramsvegen 201, N-9037 Tromsø, Norway \\ (*corresponding author, email: sabina.s.palinkas@uit.no) \\ ${ }^{2}$ University of Zagreb, Faculty of Science, Geological Department, Horvatovac 95, HR-10000 Zagreb, Croatia \\ ${ }^{3}$ Thermo Fisher Scientific, Hanna-Kunath-Straße 11, D-28199 Bremen, Germany \\ ${ }^{4}$ University of Rijeka, Medical Faculty, Department of Physics, Braće Branchetta 20, HR-51 000 Rijeka, Croatia \\ ${ }^{5}$ Institute of Nuclear Research, Hungarian Academy of Sciences, Bemtér 18/C, H-4001 Debrecen, Hungary \\ ${ }^{6}$ Prishtina University, Faculty of Mines and Metallurgy, Mitrovica, Kosovo
}
$121-142$
12 Figs.
4 Tabs.
doi: $10.4154 / g c .2016 .10$
chess

Article history:

Received September 24, 2015

Revised and accepted January 05, 2016

Avaliable online February 29, 2016
Keywords: phreatomagmatic breccia, $\mathrm{Pb}-\mathrm{Zn}$ Ag skarn deposit, transition from lithostatic to hydrostatic regime, fluid inclusions, stable isotopes, $\mathrm{K} / \mathrm{Ar}$ dating

\begin{abstract}
The Trepča $\mathrm{Pb}-\mathrm{Zn}-\mathrm{Ag}$ skarn deposit in Kosovo is spatially and temporarily related to the phreatomagmatic breccia of Oligocene age ( 23Ma). The deposit shows features typical for skarn deposits worldwide, including a stage of isochemical metamorphism, a prograde stage of an anhydrous, low oxygen and low sulfur fugacity character, and a retrograde stage characterized by an increase in the water activity as well as by an increase in oxygen and sulfur fugacities. The mineralization is hosted by the recrystallized Upper Triassic limestone. The prograde mineralization consists mainly of $\mathrm{Ca}-\mathrm{Fe}-\mathrm{Mn} \pm \mathrm{Mg}$ pyroxenes. The host recrystallized limestone at the contact with the prograde (skarn) mineralization has an increased content of $\mathrm{Fe}, \mathrm{Mn}, \mathrm{Mo}$, $\mathrm{As}, \mathrm{Au}, \mathrm{Cs}, \mathrm{Ga}, \mathrm{REE}$ and $\mathrm{Y}$ suggesting their transport by infiltrating magmatic fluids. The decreased $\delta^{13} \mathrm{C}$ and $\delta^{18} \mathrm{O}$ values reflect the contribution of magmatic $\mathrm{CO}_{2}$. The retrograde mineral assemblage comprises ilvaite, magnetite, arsenopyrite, pyrrhotite, marcasite, pyrite, $\mathrm{Ca}-\mathrm{Fe}-\mathrm{Mn} \pm \mathrm{Mg}$ carbonates and quartz. Hydrothermal ore minerals, mostly galena, sphalerite and pyrite, were deposited contemporaneously with the retrograde stage of the skarn development. Syn-ore and post-ore carbonates reflect the diminishing influence of magmatic $\mathrm{CO}_{2}$. Syn-ore carbonates are enriched in $\mathrm{Fe}, \mathrm{Mg}, \mathrm{Mn}$, many chalcophile elements, including $\mathrm{Ag}$, $\mathrm{As}, \mathrm{Bi}, \mathrm{Cd}, \mathrm{Cu}, \mathrm{Pb}, \mathrm{Sb}$ and $\mathrm{Zn}$, as well as in $\mathrm{Au}, \mathrm{Y}$ and $\mathrm{REE}$. The post-ore stage accompanied the precipitation of a significant amount of $\mathrm{Ca}$-rich carbonates including travertine deposits at the deposit surface.
\end{abstract}

The phreatomagmatic breccia was developed along a NW dipping contact between the ore bearing recrystallized limestone and the overlying schist. It has an inverted cone shape with vertical extension up to $800 \mathrm{~m}$ and a diameter of up to $150 \mathrm{~m}$. The upper part of the diatreme (an underground segment of the phreatomagmatic breccia) is characterized by the presence of a hydrothermally altered rootless quartz-latite dyke surrounded by an unsorted polymict breccia mantle. Despite the alteration processes, the dyke has a preserved porphyritic texture. Partly preserved sanidine, accompanied with a mixture of muscovite and quartz, reflects a near-neutral to weakly acidic environment. The clasts of country rocks and skarn mineralization underwent intense milling and mixing due to repeated magmatic penetrations. Sericitization of the breccia matrix, locally accompanied with minor kaolinitization, point to an increased water activity under near-neutral to weakly acidic conditions. Large fragments originally composed of anhydrous skarn minerals (pyroxenes) are usually completely altered to a mixture of fibroradial magnetite, quartz and various amounts of carbonates suggesting an increase in oxygen fugacity. Their pyrite rims reflect that the increase in oxygen fugacity was followed by an increase in sulfur fugacity. The clast predominantly composed of Fe-sulfides and minor Bi-sulfides suggest that the increase in sulfur fugacity was locally sufficient to complete sulfidation of hedenbergite to pyrrhotite and/or pyrite.

Although the phreatomagmatic breccia at the Trepča $\mathrm{Pb}-\mathrm{Zn}$-Ag skarn deposit does not carry significant amounts of ore mineralization, its formation was crucial for ore deposition. Phreatomagmatic explosions and formation of the breccia turned the system from the lithostatic to hydrostatic regime and triggered the retrograde stage increasing the water activity and oxygen fugacity in the system. In addition, cooling and decompression of the system contributed to more effective degassing of magmatic sulfur and increased the sulfur fugacity. 


\section{INTRODUCTION}

Breccia pipes have been recognized in different types of ore deposits, from porphyry to epithermal in their origin (e.g. the Acupan Au-Ag-Te epithermal deposit, Philippines (COOKE \& BLOOM, 1990); the Roşia Montană Au-Ag epithermal deposit, Romania (WALLIER et al., 2006); the Cerro de Pasco epithermal polymetallic deposit, Peru (BAUMGARTNER et al., 2008); the El Teniente $\mathrm{Cu}-\mathrm{Mo}$ porphyry deposit, Chile (CANNELL et al., 2005; VRY et al., 2010)). At some localities a genetic link between the breccia formation and ore mineralization is evident (e.g. the Wau Au deposit, Papua New

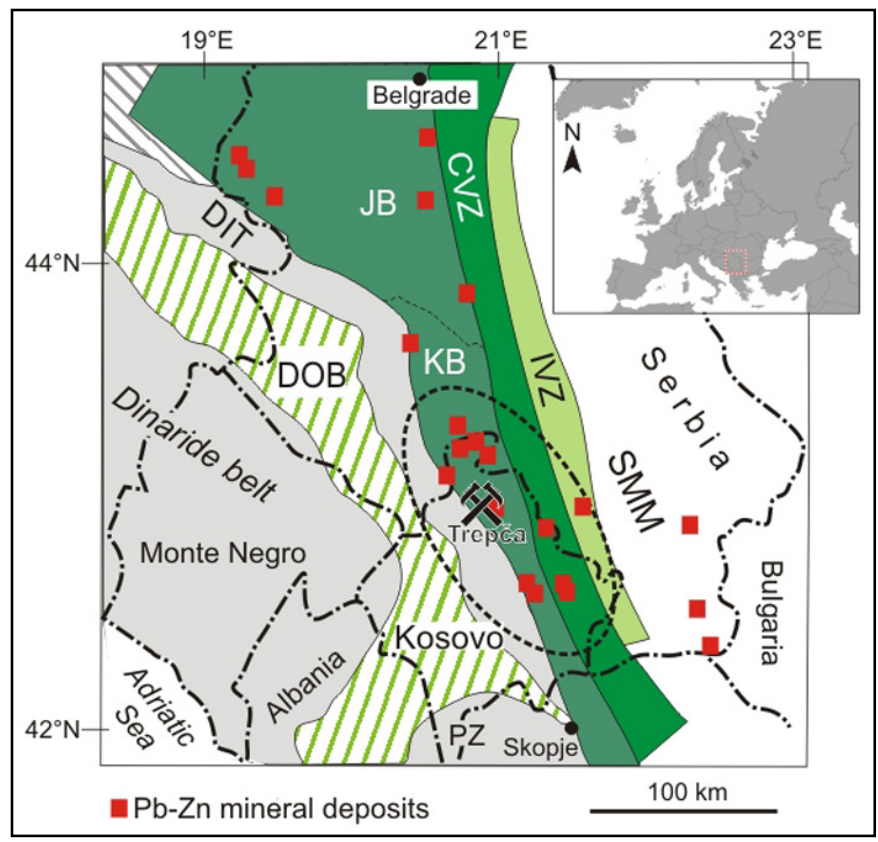

Figure 1. Geological setting of the $\mathrm{Pb}-\mathrm{Zn}$ mineral deposits, including the Trepča Pb-Zn-Ag skarn deposit, within the Vardar zone, SE Europe (simplified after KARAMATA et al. (2000) and DIMITRIJEVIĆ (2001)). External Vardar Zone: JB - Jadar Block and KB - Kopaonik Block; CVZ - Central Vardar Zone; IVZ - Internal Vardar Zone; DOB - Dinaride Ophiolite Belt; DIT - Drina-Ivanjica Terrain; PZ - Pelagonian Zone; SMM - Serbo-Macedonian Massif.
Guinea (SILLITOE et al., 1984); the Kelian Au deposit, Indonesia (DAVIES et al., 2008); the Galore Creek Cu-Au deposit, British Columbia, Canada (BYRNE \& TOSDAL, 2014)), while at others it is more obscure. Also, at some localities breccia pipes host significant amounts of ore (e.g. the Aubearing breccia pipe at Kidston, Queensland, Australia (BAKER \& ANDREW, 1991); the Cu-bearing Donoso breccia pipe, Chile (SKEWES et al., 2003); the base metal-bearing breccia pipe at the Cerro de Pasco deposit, Peru (BAUMGARTNER et al., 2008)) whereas elsewhere they are mostly barren (the Aguablanca $\mathrm{Ni}-\mathrm{Cu}$ magmatic deposit, Spain (TORNOS et al., 2001); the Copper Creek mining district, Arizona, USA (ANDERSON et al., 2009); the Blackbird Co-Cu-Au-Bi-Y-REE district, Idaho, USA (TRUMBULL et al., 2011)).

The Trepča deposit in Kosovo (Fig. 1) is an example of the $\mathrm{Pb}-\mathrm{Zn}-\mathrm{Ag}$ skarn deposit spatially and temporarily related to the phreatomagmatic breccia (STRMIĆ PALINKA $\breve{S}$ et al., 2007; FÉRAUD et al., 2007; STRMIĆ PALINKAŠ et al, 2013). The deposit, with current reserves of $31 \mathrm{Mt}$ of ore at 4.2 $\%$ of $\mathrm{Pb}, 3 \%$ of $\mathrm{Zn}$ and $86 \mathrm{~g} / \mathrm{t}$ of $\mathrm{Ag}$, together with the past production of approximately $34 \mathrm{Mt}$ of ore, represents an important source of metals in the SE part of Europe. A nearly circular breccia pipe occurs at the contact between the mineralized recrystallized limestone of Upper Triassic age and the barren schist (Fig. 2). Although the breccia does not host an economically significant amount of ore, we argue that the mechanism of its formation is a key factor for understanding ore forming processes in the Trepča deposit. The abandoned open pit and the active underground mine expose the breccia over a vertical interval of $800 \mathrm{~m}$ and allow access to various breccia lithofacies.

The major aim of this study is to understand the origin of the phreatomagmatic breccia and its role in the ore-deposition processes at the Trepča $\mathrm{Pb}-\mathrm{Zn}-\mathrm{Ag}$ skarn deposit. The study combines descriptive features, mineralogy, stable isotope and fluid inclusion data obtained from the breccia and wall rocks. The K/Ar data gained on whole rock and single grain separates revealed the age of the brecciation event.

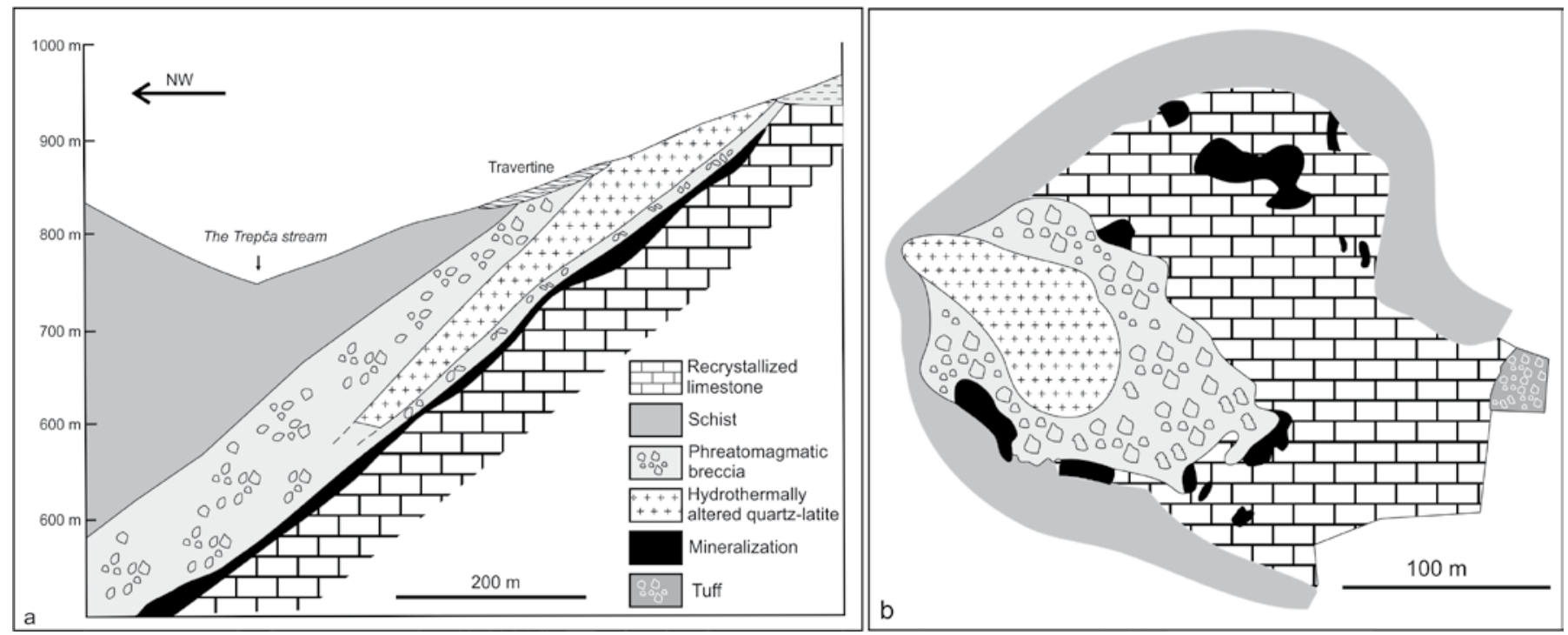

Figure 2. a. Cross section through the Trepča Pb-Zn-Ag skarn deposit; b. Local geological map of the Trepča Pb-Zn-Ag skarn deposit (after SCHUMACHER, 1950). 

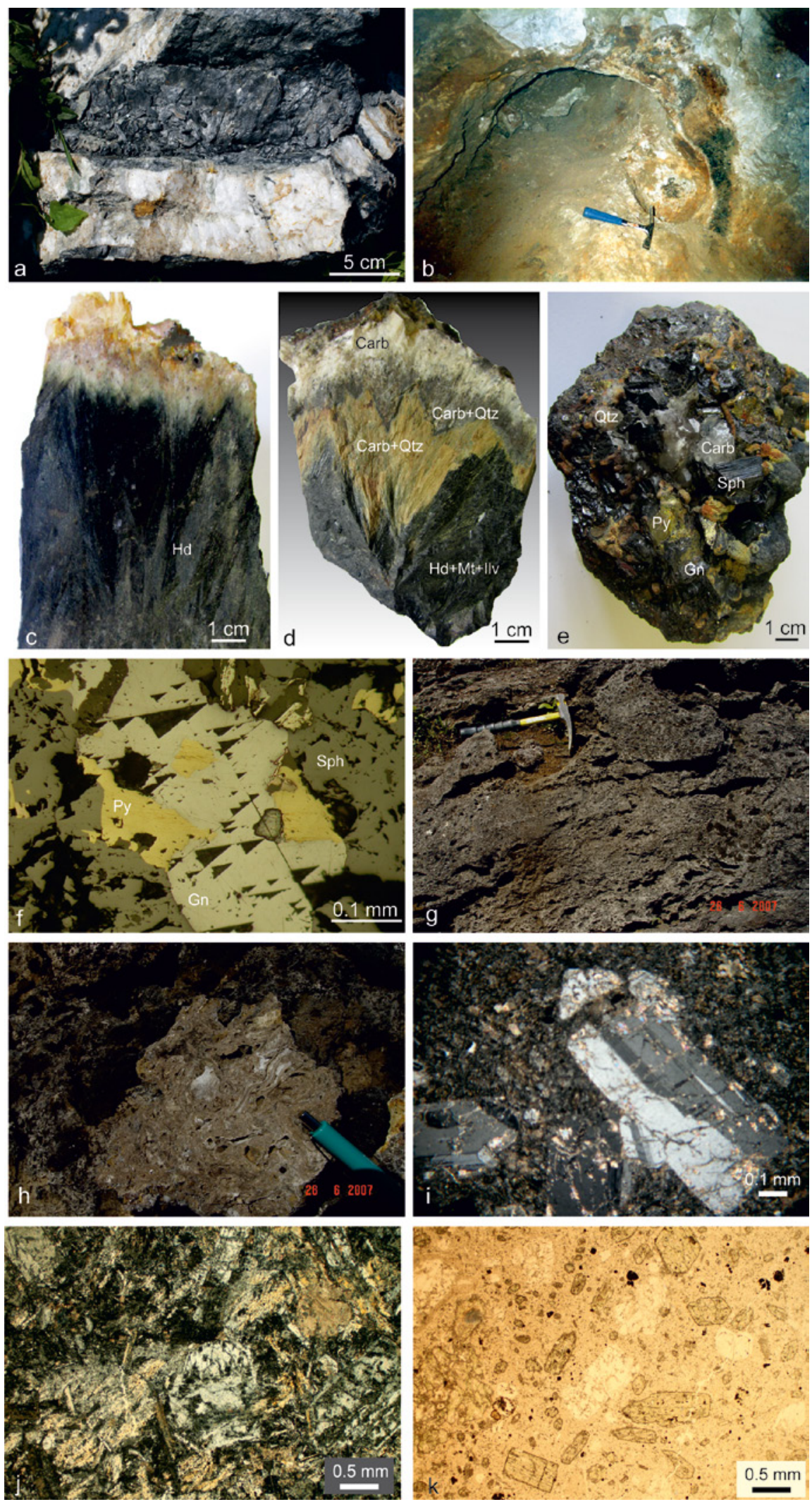

\section{GEOLOGICAL SETTING}

The Trepča $\mathrm{Pb}-\mathrm{Zn}$-Ag skarn deposit is situated in the western part of the NW-SE trending Vardar zone that extends throughout the western part of the Balkan Peninsula between the Dinarides, the Drina-Ivanjica Terrain and the Pelagonian Zone to the west and the Serbo-Macedonian Massif to the east (Fig. 1). The Vardar zone represents the main suture zone along the
Figure 3. a. Upper Palaeozoic schist intercalated with quartzite layers; b. Recrystallized Upper Triassic limestone with developed karst phenomena; c. Prograde skarn mineralization consisting mainly of pyroxenes; $d$. Retrograde skarn mineralization comprising a mixture of ilvaite, magnetite, quartz and $\mathrm{Ca}-\mathrm{Fe}-\mathrm{Mn}-\mathrm{Mg}$ carbonates; e. Hydrothermal mineralization comprising ore (galena, sphalerite, pyrite) and gangue (carbonate, quartz) minerals; $f$. The principal ore minerals, galena and sphalerite, are accompanied by various amounts of pyrite; $g$. Travertine deposits at the surface of the Trepča $\mathrm{Pb}-\mathrm{Zn}-\mathrm{Ag}$ skarn deposit (835 m above mean sea level); h. Close view of the travertine deposit; i. Photomicrographs of trachyte with typical porphyritic texture and altered sanidine phenocrysts (under crossed polarizers); j. Photomicrographs of quartz-latite with altered sanidine, plagioclases, amphiboles and quartz as principal phenocrysts (under crossed polarizers); k. Photomicrographs of andesite composed of plagioclase, amphiboles and quartz phenocrysts embedded within a carbonized and silificated aphanitic groundmass. Mineral abbreviations: Hd - hedenbergite; $\mathrm{Mt}$ magnetite, Ilv - ilvaite; Carb - Ca-Fe-Mn-Mg carbonates; Qtz - quartz; Sph - sphalerite; Gn - galena; Py - pyrite.

contact between the Adriatic and the Euroasian plate with elements of both continental and oceanic lithologies (DIMITRIJEVIĆ, 2001; KARAMATA et al., 2000; ZELIĆ et al., 2010; ROBERTSON et al., 2013).

During the Late Permian to Middle Triassic the incipient rifting process affected metamorphosed Precambrian-Palaeozoic terrains separating the Pelagonian Zone and the Serbo-Macedonian Massif. Rifting was followed by the development of a subsiding carbonate platform and formation of an oceanic crust during Late Triassic-Early Jurassic time (SHARP \& ROBERTSON, 2006; DILEK et al., 2007; ROBERTSON et al., 2013). The Western (External) Vardar Zone represents a complex zone that comprises ophiolites and ophiolitic mélange (KARAMATA et al., 1980). At several localities ophiolitic masses, composed of spinel lherzolite, harzburgite and dunite, have preserved evidence for metamorphic soles at their bases. The $\mathrm{K} / \mathrm{Ar}$ age between 160 and $123 \mathrm{Ma}$ (KARAMATA et al., 2000; MILOVANOVIĆ et al., 1995) and Ar/Ar age between 175 and $170 \mathrm{Ma}$ (BOROJEVIĆ ŚOŠTARIĆ et al., 2014) obtained from the metamorphic soles suggest emplacement of ophiolites from the Middle Jurassic to the Early Cretaceous. The Jurassic-Cretaceous mélange of the Western Vardar Zone comprises mostly large blocks and fragments of Middle to Upper Triassic and Upper Jurassic limestones, terrigenous sediments (sandstone, greywacke), basalts, and 

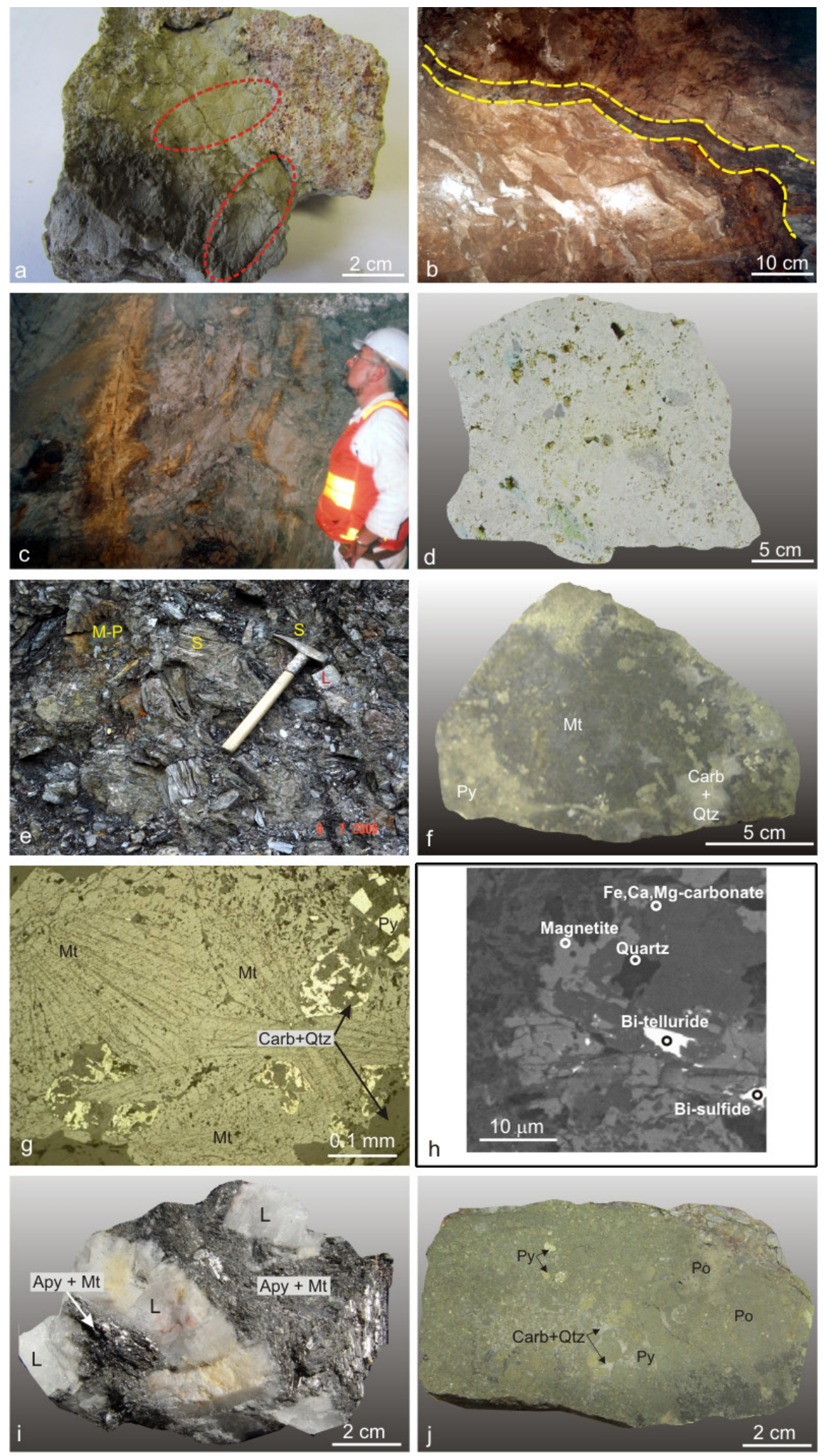

Figure 4. a. Pyroclastic rocks enclose euhedral calcite crystals, fragments of the country rocks and spheroid pumice lapillus. The leaves and plant root traces are also visible; $b$. Lateral dykelike branches of the main breccia pipe sporadically intrude the recrystallized Upper Triassic limestone; c. A gradual transition from the main breccia pipe into the brecciated schist; d. Hydrothermally altered quartz-latite from the core of the main breccia pipe. White fine-grained matrix incorporates yellowish to greenish remains of sanidine phenocrysts, quartz phenocrysts and fragments of country rocks, predominantly the schist; e. Randomly oriented fragments of schist $(\mathrm{S})$, recrystallized limestone (L) and magnetiteand pyrite-bearing clasts (M-P), the main breccia pipe, surface ( $835 \mathrm{~m}$ above mean sea level); f. Close view of the clast composed of a magnetite core and pyritic rim; g. Photomicrographs of the clast composed of a fibroradial magnetite core and pyritic rim (plane-polarized light); $h$. Scanning electron photomicrograph of the clast composed of a magnetite core and pyritic rim reveals the presence of Bi-telluride and $\mathrm{Bi}$ sulfide; i. Recrystallized limestone (L) fragment partly replaced with fibroradial arsenopyrite and magnetite (75 $\mathrm{m}$ above mean sea level); j. The clast composed of pyrite, pyrrhotite and minor Bi-sulfides. Mineral abbreviations: Mt - magnetite; Py - pyrite; Po - pyrrhotite, Apy - arsenopyrite; Carb - Ca-Fe-Mn-Mg carbonates; Qtz - quartz.

spread hydrothermal activity in the Western Vardar Zone, producing numerous skarn, hydrothermal replacement and vein type $\mathrm{Pb}-\mathrm{Zn}-\mathrm{Ag}$ deposits (Fig. 1; JANKOVIĆ, 1995; VESELINOVIĆWILLIAMS, 2011; BOROJEVIĆ ŠOŠTARIĆ et al., 2013; STRMIĆ PALINKA $\breve{S}$ et al., 2013). The Oligocene to Miocene magmatic rocks is represented mostly by trachytes, quartz-latites, andesites and pyroclastic deposits (CVETKOVIĆ et al., 2004; BOROJEVIĆ ŠOŠTARIĆ et al., 2012).

\subsection{GEOLOGY OF THE DEPOSIT}

The basement of the Trepča $\mathrm{Pb}-\mathrm{Zn}-\mathrm{Ag}$ skarn deposit comprises a metamorphosed and folded Upper Palaeozoic to Triassic sedimentary complex composed predominantly of schist and recrystallized limestone. The dark coloured schist occasiona-

cherts with Carnian to Norian and Upper Jurassic radiolarians emplaced within an argillaceous to silty matrix (SUDAR \& KOVACS, 2006; VASKOVIĆ \& MATOVIĆ, 2010). In contrast, the Eastern (Internal) Vardar Zone comprises the weakly metamorphosed mélange of Jurassic age with predominantly basaltic fragments (SUDAR \& KOVACS, 2006, and references therein).

The postcollisional magmatism of Oligocene to Miocene age (CVETKOVIĆ et al., 2004) was accompanied by wide- lly is intercalated with compact dense to coarse-grained quartzite layers (Fig. 3a). At the contact with the mineralization the schist is enriched in quartz and micas. The recrystallized limestone frequently exhibits developed karst phenomena (Fig. 3b). The size of calcite grains varies from several millimetres at places spatially distal to the mineralization up to several centimeters along the contact with the ore bodies and the breccia pipe. According to the data obtained from conodont remains the limestone has been assigned to the Upper 
Triassic (SUDAR, 1986). The limestone-schist contact is marked by the presence of the breccia pipe (SCHUMACHER, 1950, 1954; FÉRAUD et al., 2007; STRMIĆ PALINKAŠ et al., 2013).

The mineralization of the Trepča $\mathrm{Pb}-\mathrm{Zn}$-Ag skarn deposit is exclusively hosted by the recrystallized limestone (Fig. 2). The principal skarn minerals in the Trepča deposit are Ca-Fe$\mathrm{Mn} \pm \mathrm{Mg}$ silicates including pyroxenes, ilvaite and minor garnets. Accessory minerals are $\mathrm{Ca}-\mathrm{Fe}-\mathrm{Mn} \pm \mathrm{Mg}$ carbonates and quartz. Paragenetic studies based on macro- and microtextures show that the skarn assemblage from the Trepča deposit was formed in several stages, similar to other skarns (MEINERT, 1992; MALO et al., 2000; MEINERT et al., 2005; CANET et al, 2011). The prograde stage has an anhydrous character with $\mathrm{Ca}-\mathrm{Fe}-\mathrm{Mn} \pm \mathrm{Mg}$ pyroxenes as the major minerals (Fig. 3c). CaFe garnets (andradite) occur rarely, exclusively in the uppermost levels of the deposit. The retrograde stage has a predominantly hydrous character with ilvaite, magnetite, carbonate and quartz as important products (Fig. 3d). The hydrothermal ore minerals commonly overprint the pyroxene-rich calcic skarn, although skarn mineralization free of the ore assemblage as well as the ore mineralization without skarn precursor has been found (Fig. 3e). Black coloured sphalerite, galena
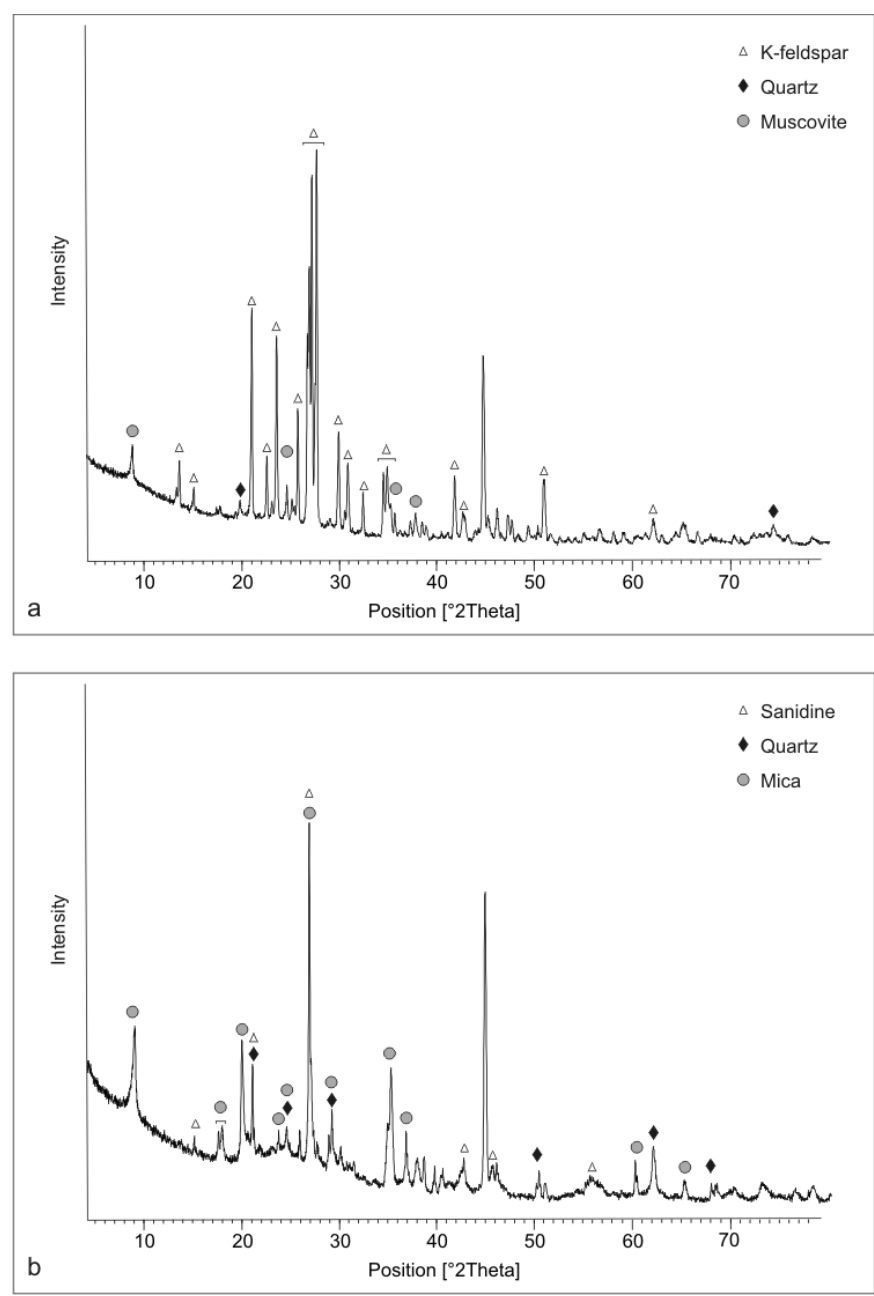

Figure 5. X-ray diffraction patterns reveal that a. hydrothermally altered quartz-latite matrix consists of K-feldspar, quartz and muscovite; b. altered phenocrysts comprise sanidine remains as well as various micas and quartz. and pyrite are the most abundant sulfide minerals (Fig. 3f). The deposit contains volumetrically minor, but mineralogically diverse Bi-bearing minerals, including native $\mathrm{Bi}$, bismuthinite $\left(\mathrm{Bi}_{2} \mathrm{~S}_{3}\right)$, cosalite $\left(\mathrm{Pb}_{2} \mathrm{Bi}_{2} \mathrm{~S}_{5}\right)$, cannizzarite $\left(\mathrm{Pb}_{4} \mathrm{Bi}_{6} \mathrm{~S}_{13}\right)$, lillianite $\left(\mathrm{Pb}_{3} \mathrm{Bi}_{2} \mathrm{~S}_{6}\right)$, ikunolite $\left(\mathrm{Bi}_{4}(\mathrm{~S}, \mathrm{Se})_{3}\right)$, babkinite $\left(\mathrm{Pb}_{2} \mathrm{Bi}_{2}(\mathrm{~S}, \mathrm{Se})_{3}\right)$, joseite $\left(\mathrm{Bi}_{4}(\mathrm{~S}, \mathrm{Te})_{3}\right)$, heyrovskyite $\left(\mathrm{Pb}_{10} \mathrm{Ag}\right.$ $\left.\mathrm{Bi}_{5} \mathrm{~S}_{18}\right)$ andizoklakeite $\left(\mathrm{Pb}_{27}(\mathrm{Cu}, \mathrm{Fe})_{2}(\mathrm{Sb}, \mathrm{Bi})_{19} \mathrm{~S}_{57}\right)$ (TERZIĆ et al., 1974; KOŁODZIEJCZYK et al., 2015). Travertine deposits locally occur as a top layer capping the deposit and marking the preserved palaeosurface (Figs. $3 \mathrm{~g}, \mathrm{~h}$ ).

During the Oligocene-Miocene, the Trepča area was a location of considerable volcanicactivity characterized by numerous lava flows and large masses of volcanoclastic rocks. The volcanic rocks are represented mostly by trachytes, quartzlatites and andesites. Trachytes are composed of a light gray matrix and sanidine phenocrysts (Fig. 3i). Quartz-latites are exposed at the Zvečan hill and occur as the core of the breccia pipe within the deposit. Beside sanidine they comprise significant amounts of quartz grains (Fig. 3j). The Ar/Ar age of the Zvečan hill quartz-latite spans between $24.8 \pm 0.2 \mathrm{Ma}$ for $\mathrm{K}$ feldspar and 25.8 $\pm 0.3 \mathrm{Ma}$ for amphibole (BOROJEVIĆ ŠOŠTARIĆ et al., 2012). Andesites are mainly light to medium gray in colour and porphyritic in texture. They are composed of plagioclase, hornblende, biotite, augite and bronzite (Fig. 3k)

\subsection{THE BRECCIA GEOMETRY, COMPOSITION AND INTERNAL ORGANIZATION}

The breccia pipe exposed at the Trepča Pb-Zn-Ag skarn has an inverted cone shape characteristic for phreatomagmatic breccias elsewhere (e.g. SILLITOE, 1985; TAMAS \& MILESI, 2002; LANDTWING et al., 2002; DAVIES et al., 2008). The diatreme (an underground segment of the phreatomagmatic breccia; LORENZ, 1973) extends vertically up to $800 \mathrm{~m}$ below the surface and has a diameter of approximately $150 \mathrm{~m}$ (Fig. 2). The partly preserved maar structure (a surface expression of phreatomagmatic breccia; LORENZ, 1973) comprises tuffaceous (Fig. 2; SCHUMACHER, 1950) and pyroclastic deposits with common remnants of plant leaves and roots (Fig. 4a). The breccia pipe was emplaced along a NW dipping contact between the ore bearing recrystallized limestone and the overlying schist (Fig. 2). Contacts between the breccia and the recrystallized limestone are mostly sharp but locally cut by lateral dyke-like branches (Fig. 4b) infilled with rock flour ("milled matrix fluidized breccia") or with angular fragments ("jigsaw-puzzle breccia"). In contrast, the contacts with the overlying schist are unclear, with a gradual transition from the breccia pipe into the brecciated schist (Fig. 4c). The upper part of the diatreme hosts a hydrothermally altered quartz-latite dyke with the preserved porphyritic texture (Fig. 4d). The white fine-grained matrix comprises muscovite, quartz, and K-feldspars (Fig. 5). The sanidine phenocrysts are partly altered to a yellowish to greenish fine-grained mixture of muscovite and quartz (Fig. 5). The quartz phenocrysts are well preserved. Fragments of county rocks, especially schist, are embedded within the matrix too. The dyke occupies the pipe core and is surrounded by the unsorted polymict breccia mantle (Figs. 2, 4e). 
Table 1. Analyzed samples from the Trepča Pb-Zn-Ag skarn deposit.

\begin{tabular}{|c|c|c|c|}
\hline Sample & Level & $\begin{array}{l}\text { Depth } \\
\text { (m a.m.s.l.*) }\end{array}$ & Description \\
\hline KM-10 & surface & 835 & $\begin{array}{l}\text { host limestone, barren, } \\
\text { recrystallized }\end{array}$ \\
\hline $\mathrm{P} 1$ & surface & 835 & pyroclastic deposit \\
\hline $\mathrm{P} 2$ & surface & 835 & pyroclastic deposit \\
\hline $\mathrm{P} 4$ & surface & 835 & travertine deposit \\
\hline TR-6 & surface & 835 & quartz-latite \\
\hline TR-108 & surface & 835 & $\begin{array}{l}\text { hydrothermally altered } \\
\text { quartz-latite core of the } \\
\text { main breccia pipe }\end{array}$ \\
\hline KM7-1 & surface & 835 & $\begin{array}{l}\text { fragment composed of } \\
\text { a magnetite core and } \\
\text { a pyrite rim, the main } \\
\text { breccia pipe }\end{array}$ \\
\hline KM8 & surface & 835 & $\begin{array}{l}\text { fragment composed of } \\
\text { a magnetite core and } \\
\text { a pyrite rim, the main } \\
\text { breccia pipe }\end{array}$ \\
\hline KM9 & surface & 835 & $\begin{array}{l}\text { fragment composed } \\
\text { of fractured pyrite, } \\
\text { pyrrhotite and minor } \\
\text { Bi-sulfides, the main } \\
\text { breccia pipe }\end{array}$ \\
\hline TR-V-1 & $\mathrm{V}$ & 375 & $\begin{array}{l}\text { hydrothermal } \\
\text { paragenesis }\end{array}$ \\
\hline TR-VII-7 & VII & 255 & $\begin{array}{l}\text { hydrothermal } \\
\text { paragenesis }\end{array}$ \\
\hline TR-VIII-2 & VIII & 195 & $\begin{array}{l}\text { hydrothermal } \\
\text { paragenesis }\end{array}$ \\
\hline TR-VIII-4 & VIII & 195 & $\begin{array}{l}\text { hydrothermal } \\
\text { paragenesis }\end{array}$ \\
\hline $\mathrm{T} 8$ & IX & 135 & $\begin{array}{l}\text { hydrothermal } \\
\text { paragenesis }\end{array}$ \\
\hline $\mathrm{T} 8 \mathrm{a}$ & IX & 135 & $\begin{array}{l}\text { hydrothermal } \\
\text { paragenesis }\end{array}$ \\
\hline $\mathrm{T} 8-2$ & IX & 135 & $\begin{array}{l}\text { hydrothermal } \\
\text { paragenesis }\end{array}$ \\
\hline STS-0 & $\mathrm{X}$ & 75 & $\begin{array}{l}\text { host limestone, } \\
\text { barren, recrystallized }\end{array}$ \\
\hline STS-1 & $\mathrm{X}$ & 75 & $\begin{array}{l}\text { hydrothermal } \\
\text { paragenesis }\end{array}$ \\
\hline STS-2 & $\mathrm{X}$ & 75 & $\begin{array}{l}\text { hydrothermal } \\
\text { paragenesis }\end{array}$ \\
\hline STS-3 & $\mathrm{X}$ & 75 & $\begin{array}{l}\text { hydrothermal } \\
\text { paragenesis }\end{array}$ \\
\hline $\mathrm{T} 1$ & $\mathrm{X}$ & 75 & $\begin{array}{l}\text { clast composed of side- } \\
\text { rite, quartz and dickite, } \\
\text { the main breccia pipe }\end{array}$ \\
\hline $\mathrm{T} 2$ & $\mathrm{X}$ & 75 & $\begin{array}{l}\text { recrystallized limestone } \\
\text { fragment, the main } \\
\text { breccia pipe }\end{array}$ \\
\hline
\end{tabular}

\begin{tabular}{|c|c|c|c|}
\hline Sample & Level & $\begin{array}{l}\text { Depth } \\
\text { (m a.m.s.l.*) }\end{array}$ & Description \\
\hline $\mathrm{T} 2$ & $\mathrm{X}$ & 75 & $\begin{array}{l}\text { recrystallized lime- } \\
\text { stone fragment partly } \\
\text { replaced by magnetite } \\
\text { and aresenopyrite, the } \\
\text { main breccia pipe }\end{array}$ \\
\hline $\mathrm{T} 3$ & $\mathrm{X}$ & 75 & $\begin{array}{l}\text { the recrystallized lime- } \\
\text { stone in contact with } \\
\text { the main breccia pipe }\end{array}$ \\
\hline $\mathrm{T} 3 \mathrm{a}$ & $\mathrm{X}$ & 75 & $\begin{array}{l}\text { the recrystallized lime- } \\
\text { stone in contact with } \\
\text { the main breccia pipe }\end{array}$ \\
\hline $\mathrm{T} 4$ & $\mathrm{X}$ & 75 & $\begin{array}{l}\text { recrystallized limestone } \\
\text { at the contact with lat- } \\
\text { eral breccia branches }\end{array}$ \\
\hline $\mathrm{T} 4 \mathrm{a}$ & $\mathrm{X}$ & 75 & lateral breccia branch \\
\hline T5 & $\mathrm{X}$ & 75 & $\begin{array}{l}\text { hydrothermal } \\
\text { paragenesis }\end{array}$ \\
\hline T6a & $\mathrm{X}$ & 75 & skarn paragenesis \\
\hline $\mathrm{T} 6 \mathrm{~b}$ & $\mathrm{X}$ & 75 & skarn paragenesis \\
\hline T6c & $\mathrm{X}$ & 75 & skarn paragenesis \\
\hline T6d & $\mathrm{X}$ & 75 & skarn paragenesis \\
\hline T6e & $\mathrm{X}$ & 75 & skarn paragenesis \\
\hline T6f & $\mathrm{X}$ & 75 & $\begin{array}{l}\text { host limestone, barren, } \\
\text { recrystallized }\end{array}$ \\
\hline $\mathrm{T} 7-2$ & $\mathrm{X}$ & 75 & $\begin{array}{l}\text { hydrothermal parage- } \\
\text { nesis }\end{array}$ \\
\hline $\mathrm{T} 7-3 \mathrm{a}$ & $\mathrm{X}$ & 75 & $\begin{array}{l}\text { hydrothermal } \\
\text { paragenesis }\end{array}$ \\
\hline $\mathrm{T} 7-4$ & $\mathrm{X}$ & 75 & $\begin{array}{l}\text { recrystallized limestone } \\
\text { at the contact with late- } \\
\text { ral breccia branches }\end{array}$ \\
\hline $\mathrm{T} 7-4 \mathrm{a}$ & $\mathrm{X}$ & 75 & lateral breccia branch \\
\hline $\mathrm{T} 7-5$ & $\mathrm{X}$ & 75 & $\begin{array}{l}\text { hydrothermal } \\
\text { paragenesis }\end{array}$ \\
\hline T9-1 & $\mathrm{X}$ & 75 & skarn paragenesis \\
\hline T9-3 & $\mathrm{X}$ & 75 & skarn paragenesis \\
\hline T9-4 & $X$ & 75 & skarn paragenesis \\
\hline T9-5 & $\mathrm{X}$ & 75 & $\begin{array}{l}\text { hydrothermal } \\
\text { paragenesis }\end{array}$ \\
\hline T9-6 & $X$ & 75 & skarn paragenesis \\
\hline $148 \mathrm{~A}$ & $X$ & 75 & $\begin{array}{l}\text { hydrothermal } \\
\text { paragenesis }\end{array}$ \\
\hline TR-XI-2 & XI & 15 & $\begin{array}{l}\text { hydrothermal } \\
\text { paragenesis }\end{array}$ \\
\hline TR-XI-3 & XI & 15 & $\begin{array}{l}\text { hydrothermal } \\
\text { paragenesis }\end{array}$ \\
\hline TR-XI-4 & XI & 15 & $\begin{array}{l}\text { hydrothermal } \\
\text { paragenesis }\end{array}$ \\
\hline TR-XI-5 & XI & 15 & $\begin{array}{l}\text { hydrothermal } \\
\text { paragenesis }\end{array}$ \\
\hline TR-XI-6 & XI & 15 & $\begin{array}{l}\text { hydrothermal } \\
\text { paragenesis }\end{array}$ \\
\hline
\end{tabular}

* a.m.s.l. - above mean sea level 
Clasts are angular to well-rounded ranging in size from less than a millimetre to several metres with no systematic distribution in fragment size and roundness. They occupy up to 90 vol.\% of the breccia. Fragments of the country rocks, limestones and schists, together with $\mathrm{Ca}-\mathrm{Fe}-\mathrm{Mn} \pm \mathrm{Mg}$ silicate-, magnetite- and sulfide-bearing fragments represent the principal types of clasts. The upper portion of the diatreme is characterized by the extreme mixing of the rock fragments of various origin but besides the country rock fragments, the fragments composed of the magnetite core and pyrite-enriched rim are the most frequent. Magnetite occurs in the form of fibroradial to spherulitic aggregates (Fig. 4f) with minor masses of pyrite, Bi-sulfides, Bi-tellurides, carbonates and quartz emplaced between magnetite grains (Figs. $4 \mathrm{~g}$, h). The rim comprises fresh pyrite accompanied by carbonates and quartz (Figs. 4f, g). Fragments of pyroclastic rocks and fragments with lacustrine plant remains have been found at various depths suggesting collapse events (McCALLUM, 1985; BAKER et al., 1986).

In the deeper part of the breccia pipe, magnetite and overprinting fibroradial arsenopyrite are found to be embedded within recrystallized limestone fragments (Fig. 4i). The clasts composed of fractured pyrite, pyrrhotite and minor Bi-sulfides are common at various depths of the breccia pipe. The space between sulfide grains is filled with a fine-grained mixture of carbonates and quartz (Fig. 4j). Quartz-latite (juvenile) fragments occur as well. The breccia matrix comprises a finegrained rock flour that has been affected by various types of hydrothermal alterations, including sericitization, kaolinitization, pyritization and carbonatization.

\section{SAMPLES AND METHODS}

A total of forty-nine hand-picked rock samples were collected from existing underground works and from the surface of the Trepča $\mathrm{Pb}-\mathrm{Zn}$-Ag skarn deposit. We sampled various types of breccia fragments, wall rocks as well as mineral parageneses (Table 1).

Paragenetic relationships were studied in thin sections by transmitted polarized light microscopy. Ore minerals were examined in polished thick sections by reflected light microscopy. X-ray powder diffraction (XRD) analysis was conducted at the University of Zagreb on a Philips PW 3040/60 X'Pert PRO powder diffractometer $(45 \mathrm{kV}, 40 \mu \mathrm{A})$, with $\mathrm{CuK} \alpha-$ monochromatized radiation $(\lambda=1.54056 \AA)$ and $\theta-\theta$ geometry. The area between 4 and $63^{\circ} 2 \theta$, with $0.02^{\circ}$ steps, was measured with a $0.5^{\circ}$ primary beam divergence. Compound identifications were based on a computer program X'Pert high score $1.0 \mathrm{~B}$ and literature data. The textural features and semiquantitative analyses of breccia fragments were examined by a Tescan Scanning Electron Microscope (SEM) equipped with an INCA 250 analyzing system and Oxford detectors at the University of Zagreb. The analyses were performed on carbon-coated polished thin sections using the following operating conditions: $3-40 \mathrm{~mm}$ beam, accelerating voltage $20 \mathrm{kV}$, current $10 \mathrm{nA}$ and counting time of 200 seconds. Bulk chemical compositions of 14 selected samples were prepared in an agate ball mill and analyzed at Acme Analytical Laboratories (Vancouver, Canada) after lithium metaborate or tetraborate fusion using inductively coupled plasma (ICP) for major elements and inductively coupled plasma-mass spectrometry (ICP-MS) for trace elements.

Microthermometric measurements of fluid inclusions within transparent minerals (calcite, quartz) were performed at the University of Zagreb. Double polished, $\sim 0.5$-mm-thick, transparent mineral wafers were used. Measurements were carried out on a Linkam THMS 600 stage mounted on an Olympus BX 51 microscope using 10× and 50× Olympus long-working distance objectives for visible light. Two synthetic fluid inclusion standards ( $\mathrm{SYN}$ FLINC; pure $\mathrm{H}_{2} \mathrm{O}$ and mixed $\mathrm{H}_{2} \mathrm{O}-\mathrm{CO}_{2}$ ) were used to calibrate the equipment. The precision of the system was $\pm 2.0^{\circ} \mathrm{C}$ for homogenization temperatures, and $\pm 0.2^{\circ} \mathrm{C}$ in the temperature range between $-60^{\circ}$ and $+10^{\circ} \mathrm{C}$. Microthermometric measurements were made on carefully defined fluid inclusion assemblages, representing groups of inclusions that were trapped simultaneously. The fluid inclusion assemblages were identified based on petrography prior to heating and freezing. If all of the fluid inclusions within the assemblage showed similar homogenization temperatures, the inclusions were assumed to have trapped the same fluid and to have not been modified by leakage or necking; these fluid inclusions thus record the original trapping conditions (GOLDSTEIN \& REYNOLDS, 1994; GOLDSTEIN, 2001; BODNAR, 2003).

Carbon and oxygen isotope analyses of carbonates from the wall rocks and breccia fragments as well as analyses of carbonates associated with the ore mineralization were performed at the University of Rijeka. Carbonate powder was extracted from hand-picked samples using a dentist's drill. A mass of $250 \mu \mathrm{g}$ of powder has been loaded in sealed reaction vessels, then flushed with helium gas and reacted at $72^{\circ} \mathrm{C}$ with phosphoric acid. The evolved carbon dioxide was sampled using a Thermo Finnigan Gas-Bench and isotope ratios were measured in continuous flow mode using a Thermo Finnigan Delta $^{\text {plus }}$ XPmass spectrometer. The data was extracted into an EXCEL file by using the ISODAT NT EXCEL export utility and further calculation steps were carried out using a predefined EXCEL worksheet. Linearity corrections were ap-

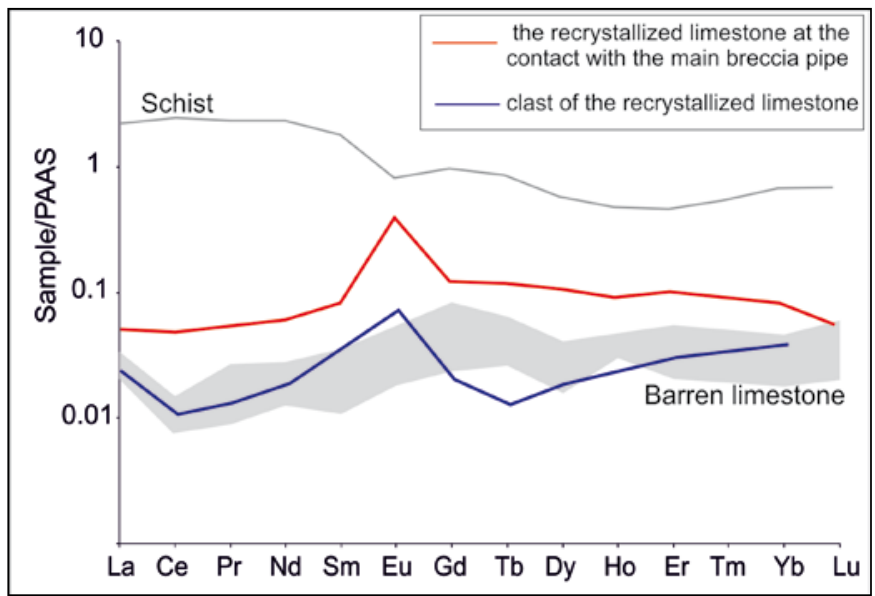

Figure 6. The post-Archean Australian shale (PAAS) normalized plots of the recrystallized limestone at the contact with the main breccia pipe and the recrystallized limestone clast from the main breccia pipe ( $75 \mathrm{~m}$ above mean sea level). The data for the schist and barren limestone are adopted from STRMIĆ PALINKAŠ et al. (2013). 
Table 2. Chemical composition of carbonates from the Trepča Pb-Zn-Ag skarn deposit.

\begin{tabular}{|c|c|c|c|c|c|c|c|c|c|c|c|c|}
\hline & & $\mathrm{SiO}_{2}$ & $\mathrm{Al}_{2} \mathrm{O}_{3}$ & $\mathrm{Fe}_{2} \mathrm{O}_{3}$ & $\mathrm{MgO}$ & $\mathrm{CaO}$ & $\mathrm{Na}_{2} \mathrm{O}$ & $\mathrm{K}_{2} \mathrm{O}$ & $\mathrm{TiO}_{2}$ & $\mathrm{P}_{2} \mathrm{O}_{5}$ & $\mathrm{MnO}$ & $\mathrm{Cr}_{2} \mathrm{O}_{3}$ \\
\hline & & \multicolumn{11}{|c|}{ ppm } \\
\hline \multicolumn{13}{|c|}{ Barren recrystallized limestone } \\
\hline STS-0* & Recrystallized limestone & 0.22 & 0.07 & 0.07 & 0.41 & 55.69 & 0.01 & 0.04 & 0.01 & 0.01 & 0.01 & 0.001 \\
\hline KM10* & Recrystallized limestone & 0.13 & 0.03 & 0.07 & 0.08 & 56.77 & 0.01 & 0.04 & 0.01 & 0.01 & 0.03 & 0.001 \\
\hline T6f* & Recrystallized limestone & 0.06 & 0.03 & 0.09 & 0.11 & 56.24 & 0.01 & 0.04 & 0.01 & 0.01 & 0.10 & 0.001 \\
\hline \multicolumn{13}{|c|}{$\begin{array}{l}\text { Recrystallized limestone at } \\
\text { contact with the breccia }\end{array}$} \\
\hline $\mathrm{T} 7-4 *$ & Contact with the milled matrix breccia & 0.14 & 0.03 & 0.09 & 0.15 & 55.91 & 0.01 & 0.04 & 0.01 & 0.01 & 0.09 & 0.001 \\
\hline $\mathrm{T}-3 \mathrm{~A}$ & Contact with the main breccia pipe & 0.35 & 0.03 & 0.54 & 0.3 & 54.37 & 0.01 & 0.04 & 0.01 & 0.03 & 0.92 & 0.001 \\
\hline \multicolumn{13}{|c|}{$\begin{array}{l}\text { Recrystallized limestone clast } \\
\text { from the breccia }\end{array}$} \\
\hline $\mathrm{T}-2$ & Recrystallized limestone clast & 0.23 & 0.08 & 0.31 & 0.34 & 55.18 & 0.01 & 0.04 & 0.01 & 0.01 & 0.27 & 0.001 \\
\hline \multicolumn{13}{|c|}{$\begin{array}{l}\text { Recrystallized limestone at con- } \\
\text { tact with the mineralization }\end{array}$} \\
\hline T6e* & $\begin{array}{l}\text { Recrystallized limestone at contact with } \\
\text { skarn mineral assemblage }\end{array}$ & 0.16 & 0.03 & 0.74 & 0.11 & 52.92 & 0.01 & 0.04 & 0.01 & 0.01 & 3.67 & 0.001 \\
\hline T9-5* & $\begin{array}{l}\text { Recrystallized limestone at contact with } \\
\text { hydrothermal mineral assemblage }\end{array}$ & 0.08 & 0.03 & 0.16 & 0.23 & 55.75 & 0.01 & 0.04 & 0.01 & 0.01 & 0.47 & 0.001 \\
\hline \multicolumn{13}{|c|}{ Syn-ore carbonates } \\
\hline T6a-1* & $\begin{array}{l}\text { Syn-ore carbonates, Skarn mineral } \\
\text { assemblage }\end{array}$ & 0.04 & 0.03 & 13.69 & 4.54 & 35.03 & 0.01 & 0.04 & 0.01 & 0.01 & 5.72 & 0.001 \\
\hline T9-5 (4)-1* & $\begin{array}{l}\text { Syn-ore carbonates, Hydrothermal } \\
\text { mineral assemblage }\end{array}$ & 0.09 & 0.03 & 9.45 & 5.79 & 34.14 & 0.01 & 0.04 & 0.01 & 0.01 & 10.32 & 0.001 \\
\hline \multicolumn{13}{|c|}{ Post-ore carbonates } \\
\hline T6a-2* & $\begin{array}{l}\text { Post-ore carbonates, Skarn mineral } \\
\text { assemblage }\end{array}$ & 0.04 & 0.03 & 0.85 & 0.23 & 53.66 & 0.01 & 0.04 & 0.01 & 0.01 & 2.46 & 0.001 \\
\hline $\mathrm{T} 7-2^{*}$ & $\begin{array}{l}\text { Post-ore carbonates, Hydrothermal } \\
\text { mineral assemblage }\end{array}$ & 0.05 & 0.03 & 0.16 & 0.22 & 54.04 & 0.01 & 0.04 & 0.01 & 0.01 & 2.88 & 0.001 \\
\hline T8 $\mathrm{a}^{*}$ & $\begin{array}{l}\text { Post-ore carbonates, Hydrothermal } \\
\text { mineral assemblage }\end{array}$ & 0.04 & 0.03 & 0.74 & 0.25 & 53.29 & 0.01 & 0.04 & 0.01 & 0.01 & 3.19 & 0.001 \\
\hline T9-5 (4)-2* & $\begin{array}{l}\text { Post-ore carbonates, Hydrothermal } \\
\text { mineral assemblage }\end{array}$ & 0.10 & 0.03 & 0.36 & 0.30 & 54.54 & 0.01 & 0.04 & 0.01 & 0.01 & 2.77 & 0.001 \\
\hline
\end{tabular}

\begin{tabular}{|c|c|c|c|c|c|c|c|c|c|c|c|c|}
\hline & & Mo & $\mathrm{Cu}$ & $\mathrm{Pb}$ & $\mathrm{Zn}$ & $\mathrm{Ni}$ & As & $\mathrm{Cd}$ & $\mathrm{Sb}$ & $\mathrm{Bi}$ & $\mathrm{Ag}$ & $\mathrm{Hg}$ \\
\hline & & \multicolumn{11}{|c|}{ ppm } \\
\hline \multicolumn{13}{|c|}{ Barren recrystallized limestone } \\
\hline STS-0* & Recrystallized limestone & $<$ d.1. & 0.9 & 5.1 & 7 & 1.3 & 0.9 & 0.2 & 0.1 & $<$ d.1. & $<$ d.1. & $<$ d.1. \\
\hline KM10* & Recrystallized limestone & $<$ d.1. & 0.3 & 6.1 & 16 & 2.1 & 1.2 & 0.2 & 0.1 & $<$ d.1. & $<$ d.1. & 0.01 \\
\hline T6 $6{ }^{*}$ & Recrystallized limestone & 0.1 & 0.3 & 6.8 & 6 & 1.7 & $<$ d.1. & 0.1 & 0.2 & $<$ d.1. & $<$ d.1. & 0.02 \\
\hline \multicolumn{13}{|c|}{$\begin{array}{l}\text { Recrystallized limestone at } \\
\text { contact with the breccia }\end{array}$} \\
\hline T7-4* & Contact with the milled matrix breccia & $<$ d.1. & 0.4 & 30.6 & 12 & 2.4 & 1.5 & 0.3 & 0.6 & $<$ d.1. & $<$ d.1. & 0.01 \\
\hline $\mathrm{T}-3 \mathrm{~A}$ & Contact with the main breccia pipe & 0.1 & 0.9 & 12.2 & 10 & 0.2 & 31.1 & 0.1 & 0.5 & 0.1 & $<$ d.1. & $<$ d.1. \\
\hline \multicolumn{13}{|c|}{$\begin{array}{l}\text { Recrystallized limestone clast } \\
\text { from the breccia }\end{array}$} \\
\hline $\mathrm{T}-2$ & Recrystallized limestone clast & 0.1 & 0.2 & 25.9 & 8 & 1.3 & 40.9 & 0.1 & 0.4 & 1.0 & 0.2 & 0.01 \\
\hline \multicolumn{13}{|c|}{$\begin{array}{l}\text { Recrystallized limestone at con- } \\
\text { tact with the mineralization }\end{array}$} \\
\hline T6e* & $\begin{array}{l}\text { Recrystallized limestone at contact with } \\
\text { skarn mineral assemblage }\end{array}$ & 0.3 & 0.2 & 6.7 & 3 & 1.0 & 2.2 & $<$ d.1. & 0.1 & $<$ d.l. & $<$ d.1. & $<$ d.1. \\
\hline $\mathrm{T} 9-5^{*}$ & $\begin{array}{l}\text { Recrystallized limestone at contact with } \\
\text { hydrothermal mineral assemblage }\end{array}$ & 0.1 & 0.4 & 7.2 & 10 & 3.4 & $<$ d.1. & 0.2 & 0.3 & $<$ d.1. & $<$ d.1. & 0.01 \\
\hline \multicolumn{13}{|c|}{ Syn-ore carbonates } \\
\hline T6a-1* & $\begin{array}{l}\text { Syn-ore carbonates, Skarn mineral } \\
\text { assemblage }\end{array}$ & 0.4 & 0.4 & 26.3 & 49 & 0.1 & 62.9 & 0.3 & 1.3 & 0.1 & $<$ d.1. & 0.01 \\
\hline T9-5 (4)-1* & $\begin{array}{l}\text { Syn-ore carbonates, Hydrothermal } \\
\text { mineral assemblage }\end{array}$ & 0.9 & 79.5 & 399.9 & 238 & $<$ d.1. & 727.0 & 1.6 & 7.9 & 0.1 & 0.8 & 0.01 \\
\hline \multicolumn{13}{|c|}{ Post-ore carbonates } \\
\hline T6a-2* & $\begin{array}{l}\text { Post-ore carbonates, Skarn mineral } \\
\text { assemblage }\end{array}$ & 0.2 & 0.1 & 15.0 & 11 & 0.4 & 6.2 & 0.1 & 0.5 & 0.1 & $<$ d.1. & 0.02 \\
\hline $\mathrm{T} 7-2 *$ & $\begin{array}{l}\text { Post-ore carbonates, Hydrothermal } \\
\text { mineral assemblage }\end{array}$ & 0.3 & 1.9 & 600.5 & 111 & 1.2 & 14.8 & 0.9 & 2.1 & $<$ d.1. & 1.1 & $<$ d.1. \\
\hline T8 $a^{*}$ & $\begin{array}{l}\text { Post-ore carbonates, Hydrothermal } \\
\text { mineral assemblage }\end{array}$ & 0.3 & 0.2 & 8.8 & 26 & 0.4 & 6.3 & 0.2 & 0.9 & $<$ d.1. & $<$ d.1. & $<$ d.1. \\
\hline T9-5 (4)-2* & $\begin{array}{l}\text { Post-ore carbonates, Hydrothermal } \\
\text { mineral assemblage }\end{array}$ & 0.2 & 0.2 & 53.3 & 60 & 0.1 & 1.2 & 0.5 & 0.2 & $<$ d.1. & $<$ d.1. & 0.01 \\
\hline
\end{tabular}

\footnotetext{
$<$ d.1. - below detection llimit
}

* - major element and REE content adopted from STRMIĆ PALINKAŠ et al. (2013) 
Table 2. Chemical composition of carbonates from the Trepča $\mathrm{Pb}-\mathrm{Zn}$-Ag skarn deposit.

\begin{tabular}{|c|c|c|c|c|c|c|c|c|c|c|c|c|}
\hline & & $\mathrm{Ba}$ & Cs & $\mathrm{Ga}$ & $\mathrm{Rb}$ & $\mathrm{Sr}$ & Th & $\mathrm{U}$ & $\mathrm{W}$ & $\mathrm{Zr}$ & $\mathrm{Y}$ & $\mathrm{La}$ \\
\hline & & \multicolumn{11}{|c|}{ ppm } \\
\hline \multicolumn{13}{|c|}{ Barren recrystallized limestone } \\
\hline STS- $0^{*}$ & Recrystallized limestone & 7.0 & $<$ d.1. & $<$ d.1. & 0.7 & 243 & 0.2 & $<$ d.1. & $<$ d.1. & 1.6 & 2.6 & 1.3 \\
\hline KM10* & Recrystallized limestone & 3.3 & $<$ d.1. & 0.8 & 0.6 & 153.1 & $<$ d.1. & $<$ d.1. & $<$ d.1. & 0.7 & 0.8 & 0.8 \\
\hline T6f* & Recrystallized limestone & 6.0 & $<$ d.1. & 1.7 & 0 & 210 & $<$ d.1. & 0.3 & $<$ d.1. & 0.8 & 0.8 & 0.8 \\
\hline \multicolumn{13}{|c|}{$\begin{array}{l}\text { Recrystallized limestone at } \\
\text { contact with the breccia }\end{array}$} \\
\hline $\mathrm{T} 7-4^{*}$ & Contact with the milled matrix breccia & 2.3 & $<$ d.1. & 0.6 & 0.7 & 332.4 & $<$ d.1. & 0.2 & $<$ d.l. & 0.5 & 0.6 & $<$ d.l. \\
\hline $\mathrm{T}-3 \mathrm{~A}$ & Contact with the main breccia pipe & 4.7 & $<$ d.1. & $<$ d.1. & 1.3 & 164.4 & $<$ d.1. & 0.1 & 0.2 & 1.0 & 4.1 & 2.1 \\
\hline \multicolumn{13}{|c|}{$\begin{array}{l}\text { Recrystallized limestone clast } \\
\text { from the breccia }\end{array}$} \\
\hline $\mathrm{T}-2$ & Recrystallized limestone clast & 7.4 & $<$ d.1. & $<$ d.l. & 0.8 & 200.8 & $<$ d.1. & $<$ d.1. & 0.3 & 1.3 & 1.2 & 0.9 \\
\hline \multicolumn{13}{|c|}{$\begin{array}{l}\text { Recrystallized limestone at con- } \\
\text { tact with the mineralization }\end{array}$} \\
\hline T6e* & $\begin{array}{l}\text { Recrystallized limestone at contact with } \\
\text { skarn mineral assemblage }\end{array}$ & 5.1 & 0.3 & 2.6 & $<$ d.1. & 64.1 & $<$ d.1. & $<$ d.l. & $<$ d.1. & $<$ d.l. & 36.9 & 28.6 \\
\hline $\mathrm{T} 9-5^{*}$ & $\begin{array}{l}\text { Recrystallized limestone at contact with } \\
\text { hydrothermal mineral assemblage }\end{array}$ & 6.1 & 0.1 & $<$ d.1. & 0.8 & 108.2 & $<$ d.1. & 0.2 & $<$ d.1. & 1.2 & 3.0 & 1.4 \\
\hline \multicolumn{13}{|c|}{ Syn-ore carbonates } \\
\hline T6a-1* & $\begin{array}{l}\text { Syn-ore carbonates, Skarn mineral } \\
\text { assemblage }\end{array}$ & 3.9 & 0.5 & 1.6 & $<$ d.1. & 104 & $<$ d.1. & 0.1 & 0.9 & $<$ d.1. & 27.4 & 17.0 \\
\hline T9-5 (4)-1* & $\begin{array}{l}\text { Syn-ore carbonates, Hydrothermal } \\
\text { mineral assemblage }\end{array}$ & 14.1 & 0.1 & 1.2 & $<$ d.l. & 120.1 & $<$ d.1. & $<$ d.1. & 0.2 & $<$ d.l. & 9.5 & 4.4 \\
\hline \multicolumn{13}{|c|}{ Post-ore carbonates } \\
\hline T6a-2* & $\begin{array}{l}\text { Post-ore carbonates, Skarn mineral } \\
\text { assemblage }\end{array}$ & 9.4 & $<$ d.1. & $<$ d.1. & $<$ d.l. & 328.3 & 0 & 0.8 & $<$ d.1. & $<$ d.l. & 4.5 & 0.9 \\
\hline $\mathrm{T} 7-2^{*}$ & $\begin{array}{l}\text { Post-ore carbonates, Hydrothermal } \\
\text { mineral assemblage }\end{array}$ & 5.2 & $<$ d.1. & $<$ d.1. & $<$ d.1. & 229.7 & 0 & 0.2 & 0.2 & $<$ d.1. & 2.9 & 0.8 \\
\hline T8 $\mathrm{a}^{*}$ & $\begin{array}{l}\text { Post-ore carbonates, Hydrothermal } \\
\text { mineral assemblage }\end{array}$ & 4.3 & $<$ d.1. & 0.6 & $<$ d.1. & 181.2 & 0 & 0.5 & $<$ d.1. & 0.5 & 5.7 & 2.1 \\
\hline T9-5 (4)-2* & $\begin{array}{l}\text { Post-ore carbonates, Hydrothermal } \\
\text { mineral assemblage }\end{array}$ & 2.9 & $<$ d.1. & 1.2 & $<$ d.1. & 316.3 & 0 & 0.3 & $<$ d.1. & $<$ d.l. & 8.3 & 2.1 \\
\hline
\end{tabular}

\begin{tabular}{|c|c|c|c|c|c|c|c|c|c|c|c|c|}
\hline & & $\mathrm{Ce}$ & $\operatorname{Pr}$ & $\mathrm{Nd}$ & $\mathrm{Sm}$ & $\mathrm{Eu}$ & $\mathrm{Gd}$ & $\mathrm{Tb}$ & Dy & Ho & Er & $\mathrm{Tm}$ \\
\hline & & \multicolumn{11}{|c|}{ ppm } \\
\hline \multicolumn{13}{|c|}{ Barren recrystallized limestone } \\
\hline STS-0* & Recrystallized limestone & 1.2 & 0.24 & 0.9 & 0.2 & $<$ d.1. & 0.39 & 0.05 & 0.18 & 0.05 & 0.16 & $<$ d.l. \\
\hline KM10* & Recrystallized limestone & 0.6 & 0.08 & $<$ d.l. & $<$ d.1. & $<$ d.1. & 0.07 & 0.01 & 0.06 & $<$ d.1. & $<$ d.1. & $<$ d.1. \\
\hline T6f* & Recrystallized limestone & $<$ d.1. & 0.06 & $<$ d.l. & $<$ d.1. & $<$ d.1. & 0.06 & 0.02 & 0.05 & $<$ d.1. & 0.05 & $<$ d.l. \\
\hline \multicolumn{13}{|c|}{$\begin{array}{l}\text { Recrystallized limestone at } \\
\text { contact with the breccia }\end{array}$} \\
\hline $\mathrm{T} 7-4 *$ & Contact with the milled matrix breccia & $<$ d.1. & 0.05 & $<$ d.l. & $<$ d.1. & $<$ d.1. & $<$ d.1. & $<$ d.1. & 0.06 & $<$ d.1. & $<$ d.1. & $<$ d.1. \\
\hline $\mathrm{T}-3 \mathrm{~A}$ & Contact with the main breccia pipe & 4.2 & 0.51 & 2.1 & 0.5 & 0.48 & 0.63 & 0.10 & 0.51 & 0.10 & 0.32 & $<$ d.1. \\
\hline \multicolumn{13}{|c|}{$\begin{array}{l}\text { Recrystallized limestone clast } \\
\text { from the breccia }\end{array}$} \\
\hline $\mathrm{T}-2$ & Recrystallized limestone clast & 0.9 & 0.12 & 0.6 & $<$ d.l. & 0.08 & 0.10 & 0.01 & 0.08 & $<$ d.l. & 0.09 & $<$ d.1. \\
\hline \multicolumn{13}{|c|}{$\begin{array}{l}\text { Recrystallized limestone at con- } \\
\text { tact with the mineralization }\end{array}$} \\
\hline T6e* & $\begin{array}{l}\text { Recrystallized limestone at contact with } \\
\text { skarn mineral assemblage }\end{array}$ & 67.7 & 8.41 & 37.0 & 8.9 & 6.50 & 8.67 & 1.24 & 7.13 & 1.19 & 3.00 & 0.35 \\
\hline T9-5* & $\begin{array}{l}\text { Recrystallized limestone at contact with } \\
\text { hydrothermal mineral assemblage }\end{array}$ & 0.5 & 0.23 & 0.9 & 0.2 & 0.14 & 0.25 & 0.05 & 0.26 & 0.08 & 0.20 & $<$ d.1. \\
\hline \multicolumn{13}{|c|}{ Syn-ore carbonates } \\
\hline T6a-1* & $\begin{array}{l}\text { Syn-ore carbonates, Skarn mineral } \\
\text { assemblage }\end{array}$ & 26.4 & 2.92 & 11.2 & 3.0 & 6.46 & 3.52 & 0.68 & 4.36 & 0.84 & 2.48 & 0.31 \\
\hline T9-5 (4)-1* & $\begin{array}{l}\text { Syn-ore carbonates, Hydrothermal } \\
\text { mineral assemblage }\end{array}$ & 7.6 & 0.92 & 3.8 & 1.3 & 3.35 & 1.70 & 0.24 & 1.41 & 0.24 & 0.59 & 0.08 \\
\hline \multicolumn{13}{|c|}{ Post-ore carbonates } \\
\hline T6a-2* & $\begin{array}{l}\text { Post-ore carbonates, Skarn mineral } \\
\text { assemblage }\end{array}$ & 1.7 & 0.20 & 1.4 & 0.3 & 0.52 & 0.54 & 0.08 & 0.50 & 0.11 & 0.41 & 0.05 \\
\hline $\mathrm{T} 7-2 *$ & $\begin{array}{l}\text { Post-ore carbonates, Hydrothermal } \\
\text { mineral assemblage }\end{array}$ & 1.2 & 0.13 & 0.8 & 0.1 & 0.26 & 0.39 & 0.05 & 0.35 & 0.08 & 0.21 & $<$ d.1. \\
\hline T8a* & $\begin{array}{l}\text { Post-ore carbonates, Hydrothermal } \\
\text { mineral assemblage }\end{array}$ & 3.1 & 0.42 & 1.8 & 0.4 & 0.50 & 0.88 & 0.11 & 0.66 & 0.14 & 0.38 & 0.06 \\
\hline T9-5 (4)-2* & $\begin{array}{l}\text { Post-ore carbonates, Hydrothermal } \\
\text { mineral assemblage }\end{array}$ & 3.2 & 0.46 & 1.9 & 0.6 & 1.25 & 1.07 & 0.18 & 1.03 & 0.24 & 0.66 & 0.08 \\
\hline
\end{tabular}


Table 2. Chemical composition of carbonates from the Trepča Pb-Zn-Ag skarn deposit.

\begin{tabular}{|c|c|c|c|c|c|}
\hline & & $\mathrm{Yb}$ & $\mathrm{Lu}$ & Sum REE & $\mathrm{Au}$ \\
\hline & & \multicolumn{3}{|r|}{ ppm } & ppb \\
\hline \multicolumn{6}{|c|}{ Barren recrystallized limestone } \\
\hline STS- $0^{*}$ & Recrystallized limestone & 0.13 & 0.03 & 4.83 & 0.5 \\
\hline KM10* & Recrystallized limestone & $<$ d.1. & $<$ d.1. & 1.62 & $<$ d.1. \\
\hline T6f* & Recrystallized limestone & 0.05 & 0.01 & 1.1 & 0.5 \\
\hline \multicolumn{6}{|c|}{$\begin{array}{l}\text { Recrystallized limestone at } \\
\text { contact with the breccia }\end{array}$} \\
\hline $\mathrm{T} 7-4 *$ & Contact with the milled matrix breccia & $<$ d.1. & $<\mathrm{d} .1$. & 0.11 & $<$ d.l. \\
\hline $\mathrm{T}-3 \mathrm{~A}$ & Contact with the main breccia pipe & 0.25 & 0.03 & 11.83 & $<$ d.l. \\
\hline \multicolumn{6}{|c|}{$\begin{array}{l}\text { Recrystallized limestone clast } \\
\text { from the breccia }\end{array}$} \\
\hline $\mathrm{T}-2$ & Recrystallized limestone clast & 0.11 & $<$ d.1. & 2.99 & 1.0 \\
\hline \multicolumn{6}{|c|}{$\begin{array}{l}\text { Recrystallized limestone at con- } \\
\text { tact with the mineralization }\end{array}$} \\
\hline T6e* & $\begin{array}{l}\text { Recrystallized limestone at contact with } \\
\text { skarn mineral assemblage }\end{array}$ & 1.69 & 0.25 & 180.63 & 1.1 \\
\hline $\mathrm{T} 9-5^{*}$ & $\begin{array}{l}\text { Recrystallized limestone at contact with } \\
\text { hydrothermal mineral assemblage }\end{array}$ & 0.13 & 0.03 & 4.37 & $<$ d.l. \\
\hline \multicolumn{6}{|c|}{ Syn-ore carbonates } \\
\hline T6a-1* & $\begin{array}{l}\text { Syn-ore carbonates, Skarn mineral } \\
\text { assemblage }\end{array}$ & 2.03 & 0.23 & 81.43 & 3.9 \\
\hline T9-5 (4)-1* & $\begin{array}{l}\text { Syn-ore carbonates, Hydrothermal } \\
\text { mineral assemblage }\end{array}$ & 0.51 & 0.06 & 26.2 & 16.6 \\
\hline \multicolumn{6}{|c|}{ Post-ore carbonates } \\
\hline T6a-2* & $\begin{array}{l}\text { Post-ore carbonates, Skarn mineral } \\
\text { assemblage }\end{array}$ & 0.28 & 0.03 & 7.02 & 1.1 \\
\hline $\mathrm{T} 7-2 *$ & $\begin{array}{l}\text { Post-ore carbonates, Hydrothermal } \\
\text { mineral assemblage }\end{array}$ & 0.15 & 0.03 & 4.55 & $<$ d.l. \\
\hline T8a* & $\begin{array}{l}\text { Post-ore carbonates, Hydrothermal } \\
\text { mineral assemblage }\end{array}$ & 0.30 & 0.05 & 10.9 & $<$ d.l. \\
\hline T9-5 (4)-2* & $\begin{array}{l}\text { Post-ore carbonates, Hydrothermal } \\
\text { mineral assemblage }\end{array}$ & 0.53 & 0.08 & 13.38 & 1.1 \\
\hline
\end{tabular}

$<$ d.1. - below detection llimit

* - major element and REE content adopted from STRMIĆ PALINKAŠ et al. (2013)

plied based on the relationships between the intensity of the first sample peak $(\mathrm{m} / \mathrm{z}=44)$ and $\delta^{18} \mathrm{O}$ values of the standards. Due to calibration based directly on the Carrara marble standard, which were part of each run, correction for calcite runs was unnecessary. The stable carbon and oxygen isotope ratios are reported in the delta (d) notation as per mil (\%o) deviation relative to the Vienna Standard Mean Ocean Water (V-SMOW) for oxygen and Vienna Pee Dee Belemnite (V-PDB) for carbon. The analytical precision was better than $\pm 0.05 \%$ or for $\delta^{13} \mathrm{C}$ and $\pm 0.1 \%$ for $\delta^{18} \mathrm{O}$.

$\mathrm{K} / \mathrm{Ar}$ dating was undertaken at the Institute of Nuclear Research of the Hungarian Academy of Science (ATOMKI), Debrecen. The whole rock samples were crushed to 0.063-0.315 $\mathrm{mm}$ size. Sanidine phenocrysts were hand-picked. One aliquot of samples (100 mg) was pulverized for potassium determination. Powders were digested in HF with the addition of some sulfuric and perchloric acids. The digested samples were dissolved in $100 \mathrm{ml} 0.25 \mathrm{~mol} / 1 \mathrm{HCl}$ and diluted fivefold. $\mathrm{Na}$ and Li (100 ppm) were added as buffer and internal standard. The potassium concentration was measured with a digitalized flame photometer. Another aliquot of samples (500 mg) was used for Ar analyses. The samples were degassed by highfrequency induction heating, and the conventional getter materials were used for cleaning Ar. The ${ }^{38} \mathrm{Ar}$ spike was intro- duced to the system from a gas pipette before degassing. The cleaned Ar was directly introduced into the mass spectrometer, operated in the static mode. Recording and evolution of Ar spectra was controlled by a microcomputer. Details of the instruments, the applied methods and results of calibration have been described by BALOGH (1985).

\section{RESULTS}

\subsection{GEOCHEMISTRY OF CARBONATES}

The major element and REE contents of carbonates from the Trepča deposit are given in STRMIĆ PALINKAŠ et al. (2013). In this publication we refer to those data and present new unpublished trace element data obtained on the same set of samples (Table 2). In addition, the recrystallized limestone from the contact with the breccia pipe and a fragment of recrystallized limestone hosted by the breccia has been analyzed (Table 2).

The barren recrystallized Upper Triassic limestone (samples STS-0, KM10 and $\mathrm{T} 6 \mathrm{f})$ is characterized by a high $\mathrm{CaO}$ content ( $\sim 56 \mathrm{wt} . \%)$ and a low content of aluminosilicate impurities $\left(\mathrm{SiO}_{2}<0.22\right.$ wt. $\%, \mathrm{Al}_{2} \mathrm{O}_{3}<0.07$ wt. \%). Large-ionlithophile elements display low concentrations with an expected exception of Sr (210-332 ppm). The REE concentrations are mostly below their detection limits, precluding estimation of complete REE patterns for this group of carbonates.

The recrystallized limestone in contact with the breccia pipe (T3a) shows an increase in $\mathrm{MnO}\left(0.9\right.$ wt. \%), $\mathrm{Fe}_{2} \mathrm{O}_{3}(0.5$ wt. \%), $\mathrm{SiO}_{2}$ (0.35 wt. \%), As (31.1 ppm), $\mathrm{Pb}$ (12.2 ppm), Y (4.1 ppm), Sb (0.5 ppm), W (0.2 ppm) and REE content $\left(\mathrm{S}_{\mathrm{REE}}\right.$ $=11.8 \mathrm{ppm})$. It exhibits the roof-shaped PAAS-normalized REE concentration pattern with a very negative $\mathrm{Ce}_{\mathrm{N}}$ and a strong positive $\mathrm{Eu}_{\mathrm{N}}$ anomaly (Fig. 6). The chemical composition of the recrystallized limestone in contact with the lateral branches of the main breccia pipe (T7-4) shows overlapping with the values obtained for the barren recrystallized limestone. However, some trace elements, including $\mathrm{Pb}(30.6$ ppm), Ni (2.4 ppm), As (1.5 ppm), Cd (0.3 ppm), and Sb (0.6 $\mathrm{ppm})$ are more abundant than in the barren limestone. Whereas the majority of REE have concentrations below their detection limits, the REE pattern cannot be plotted. The fragments of recrystallized limestone hosted by the breccia (T2) are slightly enriched in $\mathrm{MnO}(0.3$ wt. $\%), \mathrm{Fe}_{2} \mathrm{O}_{3}(0.3$ wt. \%), $\mathrm{Pb}$ (25.9 ppm), As (40.9 ppm), Sb (0.4 ppm), Ag (0.2 ppm), Au ( $1 \mathrm{ppb}), \mathrm{Tl}(0.1 \mathrm{ppm})$, and $\mathrm{W}$ content $(0.3 \mathrm{ppm})$ comparing to the barren recrystallized Upper Triassic limestone. The complete REE pattern cannot be estimated due to concentrations of Sm, Dy, Tm and Lu below their detection limits, the ob- 


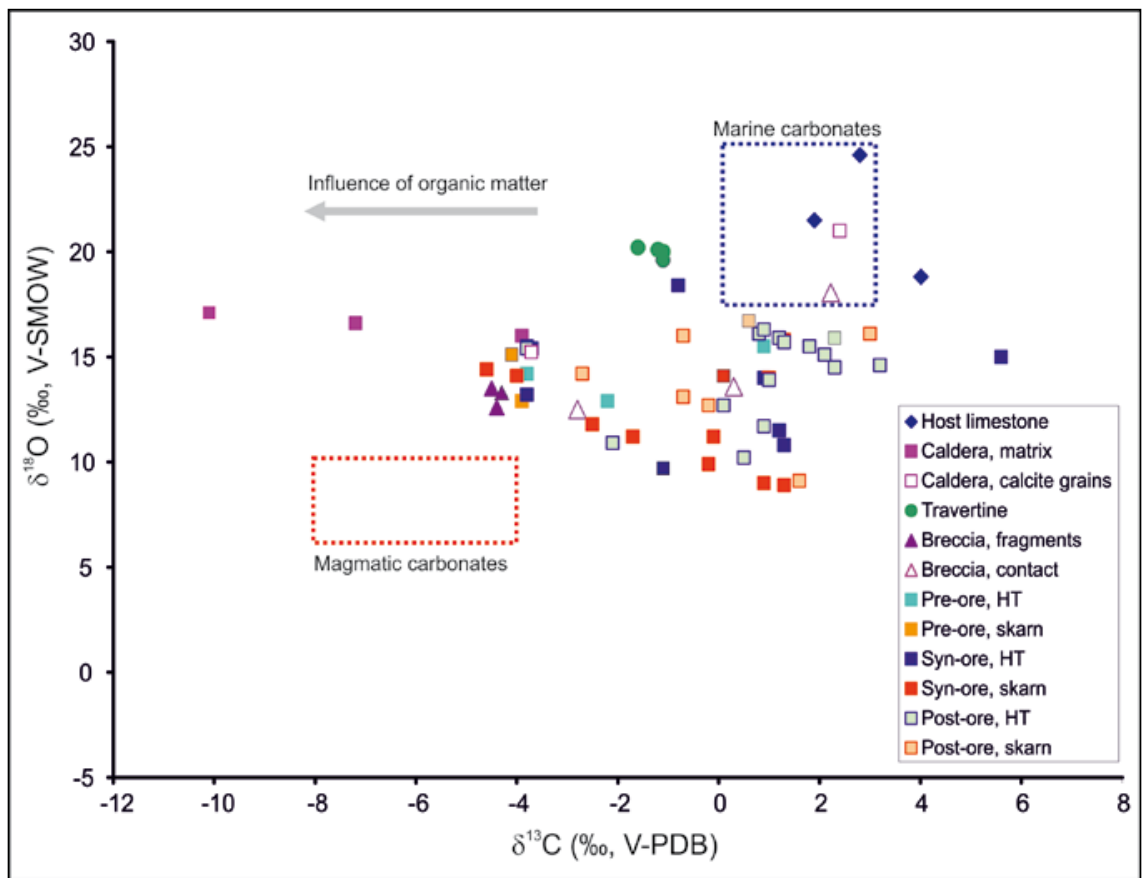

Figure 7. $\delta^{13} \mathrm{C}$ vs. $\delta^{18} \mathrm{O}$ plot of various carbonates from the Trepča Pb-Zn-Ag skarn deposit.

tained data suggest an enrichment in LREE over HREE, a week negative $\mathrm{Ce}_{\mathrm{N}}$ and a prominent positive $\mathrm{Eu}_{\mathrm{N}}$ anomaly (Fig. 6). The recrystallized limestone in contact with the skarn mineralization (T6e) is enriched in $\mathrm{MnO}\left(3.7\right.$ wt. \%), $\mathrm{Fe}_{2} \mathrm{O}_{3}$ (0.7 wt. \%), Y (36.9 ppm), REE ( $\left.\mathrm{S}_{\mathrm{REE}}=180.6 \mathrm{ppm}\right), \mathrm{Au}(1.1$ $\mathrm{ppb})$ and Mo (0.3 ppm). Concentrations of chalcophile elements are mostly within the same ranges as those obtained for the barren recrystallized limestone. Exceptions are As and Ga that show a slight increase. A decrease in Sr concentration positively correlates with a decrease in $\mathrm{Ca}$ content. The recrystallized limestone in contact with the hydrothermal mineralization (T9-6) is negligibly enriched in $\mathrm{Mn}$ and Fe comparedto the barren recrystallized Upper Triassic limestone (Table 2). Syn-ore carbonates (T6a-1, T9-5(4)-1) are significantly enriched in $\mathrm{Fe}$ (9.5-13.7 wt. \% $\left.\mathrm{Fe}_{2} \mathrm{O}_{3}\right), \mathrm{Mg}$ (4.5-5.8 wt. $\% \mathrm{MgO}$ ) and $\mathrm{Mn}$ (5.7-10.3 wt. \% MnO). Large-ion-lithophile elements display low concentrations with the expected exception for $\mathrm{Sr}$ (104-120 ppm). Caesium is slightly increased in syn-ore carbonates associated with the skarn mineral assemblage. In contrast, syn-ore carbonates from the hydrothermal mineral assemblage are enriched in Ba (Table 2). Concentrations of high field strength elements are mostly below their detection limits, with an exception for $\mathrm{Y}$ and REE that are significantly increased compared to the barren recrystallized Upper Triassic limestone (Table 2). Syn-ore carbonates are enriched in chalcophile elements, including $\mathrm{Ag}$ (up to 0.8 ppm), As (62.9-727 ppm), Bi (0.1 ppm), Cd (0.3-1.6 ppm), Cu (0.4-79.5 ppm), $\mathrm{Pb}$ (26.3-400 ppm), Sb (1.3-7.9 ppm) and $\mathrm{Zn}$ (49-238 ppm), as well as in Au (3.9-16.6 ppb). Post-ore carbonates (T6a-2, T7-2, T8a, T9-5(4)-2) are depleted in Fe (0.20.9 wt. $\left.\% \mathrm{Fe}_{2} \mathrm{O}_{3}\right), \mathrm{Mg}(0.2-0.3$ wt. $\% \mathrm{MgO}), \mathrm{Mn}(2.4-3.2$ wt. $\% \mathrm{MnO}$ ) and the majority of chalcophile elements compared to syn-ore carbonates (Table 2). However, some post-ore carbonates may have increased concentrations of $\mathrm{Pb}(\sim 600 \mathrm{ppm})$, Zn ( 110 ppm) and Ag ( 1 ppm).

\subsection{STABLE ISOTOPE COMPOSITION OF CARBONATES}

The $\delta^{18} \mathrm{O}$ and $\delta^{13} \mathrm{C}$ data obtained on the 68 carbonates from 34 hand specimens are listed in Table 3 and shown in Figure 7. The barren limestones have average $\delta^{13} \mathrm{C}$ and $\delta^{18} \mathrm{O}$ values of $2.9 \pm 1.1 \%$ V-PDB and $21.9 \pm 2.5 \%$ V-SMOW, respectively. The limestones in contact with the breccia together with the limestone fragments from the breccia are shifted toward significantly lower $\delta^{18} \mathrm{O}$ values. Recrystallized limestones in contact with the skarn $\left(\delta^{13} \mathrm{C}=-4.0 \pm 0.1 \%\right.$ o, $\delta^{18} \mathrm{O}=14.0 \pm 1.5 \%$ o and hydrothermal mineralization $\left(\delta^{13} \mathrm{C}=0.8 \pm 2.8 \%\right.$, $\delta^{18} \mathrm{O}=$ $18.5 \pm 4.2 \%$ ) differ in both their carbon and oxygen, isotopic composition. Syn-ore and post-ore carbonates exhibit depletion in ${ }^{13} \mathrm{C}$ and ${ }^{18} \mathrm{O}$ compared to the barren recrystallized limestone. Carbonates separated from the pyroclastic rocks of the caldera display variable $\delta^{13} \mathrm{C}$ and $\delta^{18} \mathrm{O}$ composition with values between -10 and $-1 \%$ and 15.5 to $-21 \%$, respectively. Travertine deposits have uniform isotopic composition with average $\delta^{13} \mathrm{C}$ and $\delta^{18} \mathrm{O}$ values of $-1.2 \pm 0.2 \%$ and $20.0 \pm 0.3 \%$, respectively.

\subsection{FLUID INCLUSION DATA}

Fluid inclusions have been studied in transparent minerals, calcite and quartz, hosted by different types of breccia fragments and in the recrystallized limestone at its contacts with the breccia (Fig. 8). The data are summarized in Figure 9.

Calcite grains selected from the recrystallized limestone at the contact with the main breccia pipe (T3a, 75 m above mean sea level) host fluid inclusions of pseudosecondary and secondary origin. According to their petrographic features, three types of fluid inclusion assemblages have been distinguished: Type 1) Assemblages composed of two phase, L-rich inclusions (Fig. 8a); Type 2) Assemblages that contain coexisting L-rich and V-rich inclusions (Fig. 8b); and Type 3) Assemblages of two phase, V-rich inclusions (Fig. 8c). Liquid-rich 

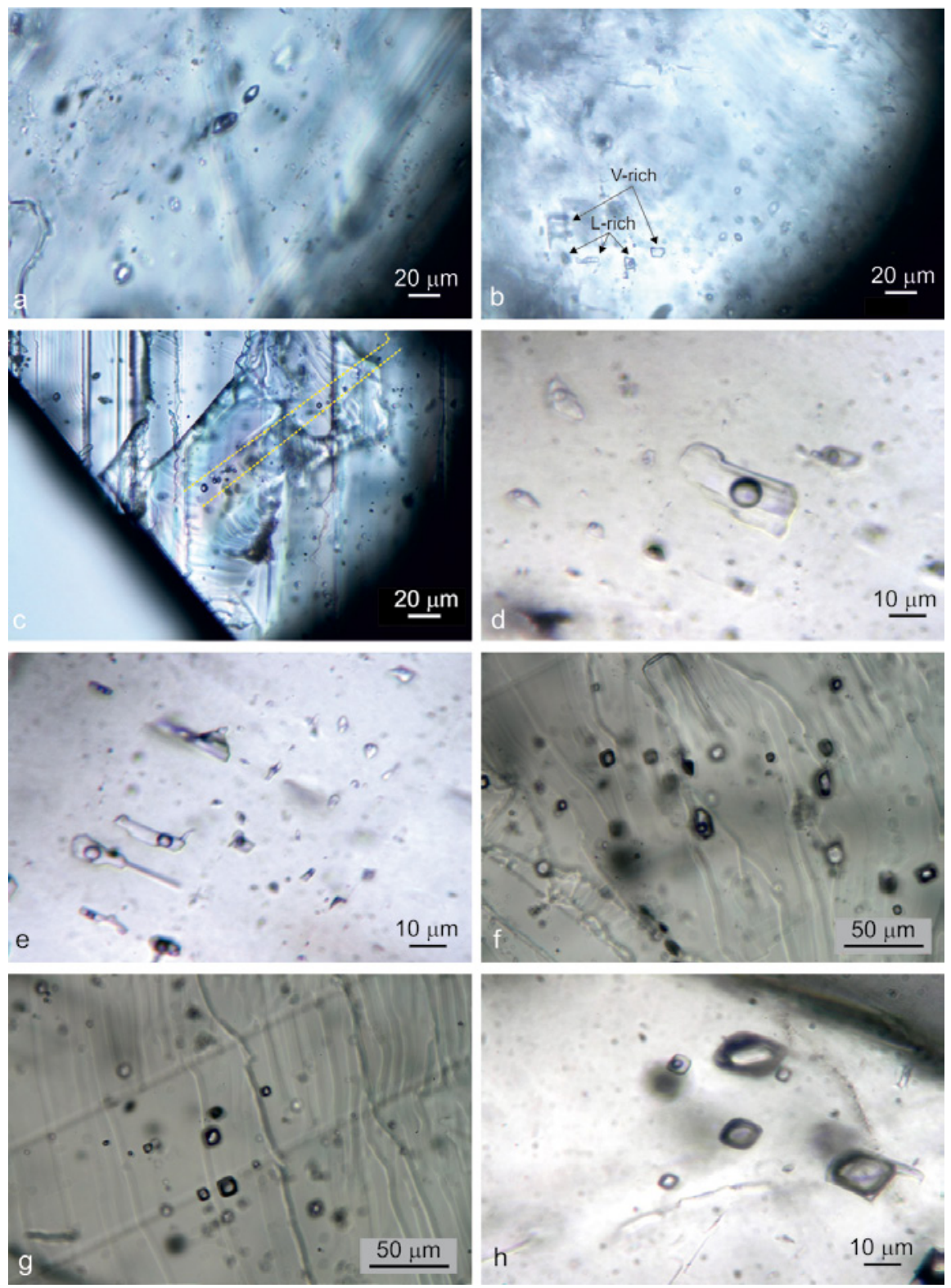

fluid inclusion assemblages (Type 1) can be subdivided into two subtypes according to their microthermometric data. Subtype 1a comprises L-rich inclusions with the degree of fill around 0.7 and homogenization temperatures $\left(\mathrm{T}_{\mathrm{h}}\right)$ between 343 and $370^{\circ} \mathrm{C}$ (Fig. 9). They have a eutectic temperature $\left(\mathrm{T}_{\mathrm{e}}\right)$ near $-50^{\circ} \mathrm{C}$ suggesting $\mathrm{CaCl}_{2}, \mathrm{NaCl}$ and $\mathrm{KCl}$ as the principal salts dissolved in the aqueous solution (BORISENKO, 1977; SAMSON \& WALKER, 2000). The salinity in the range between 19.8 to 20.6 wt.\% $\mathrm{NaCl}$ equ. (Fig. 9) is estimated from the final ice melting temperature $\left(\mathrm{T}_{\mathrm{m} \text { ice }}\right)$ recorded in the interval between -16.5 and $-17.5^{\circ} \mathrm{C}$ (BODNAR, 1993). Subtype $1 \mathrm{~b}$ comprises L-rich inclusions with the degree of fill around 0.8 and $\mathrm{T}_{\mathrm{h}}$ recorded between 275 and $290^{\circ} \mathrm{C}$ (Fig. 9). Eutectic temperatureswere recorded around $-50^{\circ} \mathrm{C}$ as well, but salinities in the range between 2.6 and $4.2 \mathrm{wt} . \% \mathrm{NaCl}$ equ. $\left(\mathrm{T}_{\mathrm{m} \text { ice }}=-1.5\right.$ to $\left.-2.5^{\circ} \mathrm{C}\right)$ are significantly lower than the values obtained for the former subtype of L-rich inclusions (Fig. 9). Fluid inclusion assemblages comprising coexisting L-rich and V-rich inclusions (Type 2) suggest an entrapment of boiling fluids (BODNAR et al., 1985). Liquid-rich inclusions have a
Figure 8. a. Pseudosecondary L-rich fluid inclusion assemblages from the recrystallized limestone at the contact with the main breccia pipe; b. Pseudosecondary coexisting L- and V-rich fluid inclusions from the recrystallized limestone at the contact with the main breccia pipe; c. Pseudosecondary V-rich fluid inclusion assemblages from the recrystallized limestone at the contact with the main breccia pipe; d. Pseudosecondary L-rich fluid inclusion assemblages from the recrystallized limestone at the contact with the lateral breccia branches; e. Pseudosecondary L-rich fluid inclusion assemblages from calcite grains in clasts composed of a magnetite core and a pyrite rim; f. Boiling effect within calcite grains separated from clasts composed of a magnetite core and a pyrite rim; g. Vapour-rich inclusions in calcite grains selected from pyroclastic deposits; h. Two-phase aqueous fluid inclusions in calcite grains selected from pyroclastic deposits.

variable degree of fill, varying between 0.6 up to 0.8 . The $\mathrm{T}_{\mathrm{e}}$ near $-50^{\circ} \mathrm{C}$ was recorded in several fluid inclusions revealing $\mathrm{CaCl}_{2}$, $\mathrm{NaCl}$ and $\mathrm{KCl}$ as the principal dissolved salts. Whereas this type of fluid inclusion has a salinity greater than 23 wt. $\% \mathrm{NaCl}$ equ., the ice dissolution precedes the hydrohalite dissolution. In some inclusions hydrohalite melts as the final solid phase. The final melting temperature in the interval between -3.2 and $0{ }^{\circ} \mathrm{C}$ suggests salinities between 25.8 and 26.2 wt. $\% \mathrm{NaCl}$ equ. (Fig. 9; STERNER et al., 1988). In other inclusions of this type, hydrohalite was transformed into halite at temperatures around $0^{\circ} \mathrm{C}$. Halite dissolves at temperatures up to $7^{\circ} \mathrm{C}$ pointing to the maximum salinity of $26.3 \mathrm{wt} \% \mathrm{NaCl}$ equ. (STERENER et al., 1988). Total homogenization occurs by vapour phase disappearance in the temperature range between 285 and $305^{\circ} \mathrm{C}$ (Fig. 9). Phase transitions in V-rich inclusions have not been recorded. Fluid inclusion assemblages that contain only V-rich inclusions (Type 3) have been recorded as well but the ambiguous phase transitions preclude their interpretation.

The recrystallized limestone at the contact with lateral branches filled with the milled matrix fluidized breccia (T4, T7-4) hosts visible fluid inclusions only in calcite grains very close to the breccia-limestone contact. Already at $5 \mathrm{~cm}$ from the contact inclusions become too small for reliable measurements $(<5 \mu \mathrm{m})$. At a distance of $10 \mathrm{~cm}$ from the contact fluid inclusions have not been detected. Measurable fluid inclusions are L-rich, with the degree of fill around 0.7 to 0.8 (Fig. 8d). Homogenization into the liquid phase was recorded in the temperature interval from 360 to $375^{\circ} \mathrm{C}$ (Fig. 9). Ice melting occurred between -7.4 and $-9^{\circ} \mathrm{C}$ corresponding to the salinity of 11.0 to 12.9 wt.\% $\mathrm{NaCl}$ equ. (Fig. 9).

The clasts that comprise a magnetite core and a pyrite rim (KM7-1, KM8) were sampled from the surface ( $835 \mathrm{~m}$ above 


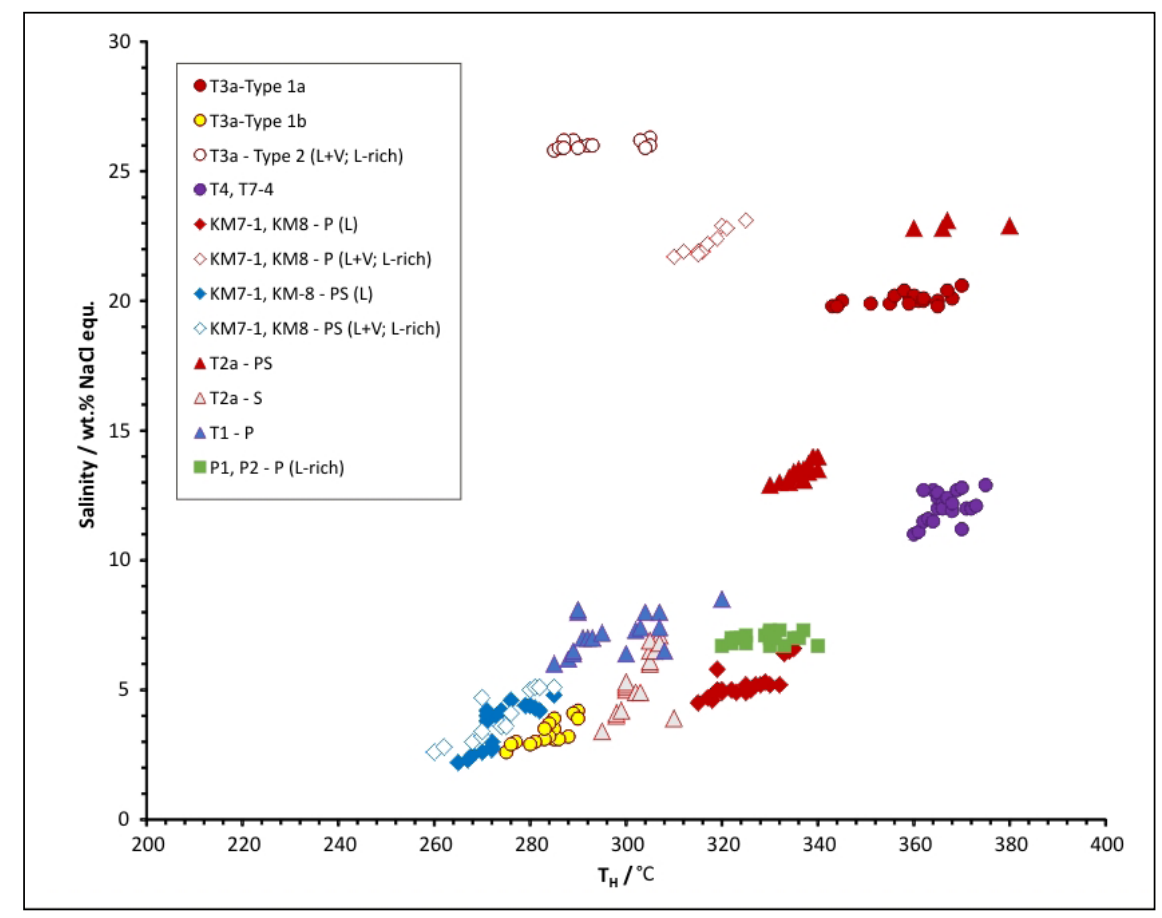

Figure 9. Correlation of homogenization temperature (TH) and salinity for fluid inclusions hosted at contacts of the phreatomagmatic breccia and its host rocks as well as within different types of clasts and pyroclastic rocks.

mean sea level). Over $80 \%$ of the primary and pseudosecondary fluid inclusions within calcite crystals belong to the two-phase, L-rich, type. They vary in size up to $25 \mathrm{~mm}$ (Fig. $8 \mathrm{e}$ ). Primary fluid inclusion assemblages homogenized into the liquid phase in the interval between 315 and $335^{\circ} \mathrm{C}$ (Fig. 9). The first melting (near-eutectic) temperature around -52 ${ }^{\circ} \mathrm{C}$ suggests that $\mathrm{CaCl}_{2}, \mathrm{NaCl}$ and $\mathrm{KCl}$ are the principal dissolved salts (BORISENKO, 1977; SAMSON \& WALKER, 2000). The $T_{m \text { hyd }}$ value recorded in the range between -27.7 and $-29.1^{\circ} \mathrm{C}$ coincides with the $\mathrm{NaCl} /\left(\mathrm{NaCl}+\mathrm{CaCl}_{2}\right)$ mass ratio between 34 and $41 \%$. The $\mathrm{T}_{\mathrm{m} \text { ice }}$ in the range between -2.7 and $-4.1{ }^{\circ} \mathrm{C}$ corresponds to an apparent salinity of4.5 to 6.6 wt.\% $\mathrm{NaCl}$ equ. (Fig. 9). Locally, the boiling effect is perceived. It is characterized by fluid inclusion assemblages comprising coexisting liquid- and vapour-rich fluid inclusions (Fig. 8f) with overlapping values of homogenization temperatures between 310 and $325^{\circ} \mathrm{C}$ (Fig. 9). Liquid-rich inclusions have relatively high salinities $\left(\mathrm{T}_{\mathrm{m} \text { ice }}=-19.1\right.$ to $-21.0^{\circ} \mathrm{C}$; salinity $=21.7$ to 23.1 wt. $\% \mathrm{NaCl}$ equ.; Fig. 9). Pseudosecondary fluid inclusion assemblages mostly comprise liquid-rich fluid inclusions with homogenization temperatures between 265 and $285^{\circ} \mathrm{C}$ (Fig. 9). Their apparent salinity ranges between 2.2 and 4.8 wt.\% $\mathrm{NaCl}$ equ. (Fig. 9; $\mathrm{T}_{\mathrm{m} \text { ice }}=-1.3$ to $-2.9^{\circ} \mathrm{C}$ ). In addition, several fluid inclusion assemblages composed of coexisting liquid- and vapour-rich inclusions have been recorded. Their homogenization temperatures mostly overlap with those obtained for the liquidrich pseudosecondary fluid inclusion assemblages, but they have slightly greater salinities (Fig. 9; $\mathrm{T}_{\mathrm{m} \text { ice }}=-1.5$ to $-3.2^{\circ} \mathrm{C}$; salinity $=2.6$ to $5.1 \mathrm{wt} . \% \mathrm{NaCl}$ equ.).

In the deeper part of the breccia, at horizon X (75 m above mean sea level), several different types of fragments were studied. Microthermometry on the majority of barren recrys- tallized limestone fragments (T2) was hampered, due to the presence of only monophase fluid inclusions. In contrast, recrystallized limestone fragments accompanied with fibroradial arsenopyrite and magnetite (T2a) have been eligible for microthermometric studies. Pseudosecondary fluid inclusion assemblages are overprinted by secondary fluids. Both fluid inclusion generations are characterized by $\mathrm{T}_{e}$ around $-50^{\circ} \mathrm{C}$ $\left(\mathrm{CaCl}_{2}-\mathrm{NaCl} \pm \mathrm{KCl}-\mathrm{H}_{2} \mathrm{O}\right.$ system $)$. Pseudosecondary fluid inclusions show $\mathrm{T}_{\mathrm{mhd}}$ in the range between -27.0 and $-28.5^{\circ} \mathrm{C}$ indicating the $\mathrm{NaCl} /\left(\mathrm{NaCl}+\mathrm{CaCl}_{2}\right)$ mass ratio between 37 and $45 \%$. The majority of fluid inclusions have moderate salinities in the range between 12.9 and $14.0 \mathrm{wt} \% \mathrm{NaCl}$ equ. (Fig. 9; $\mathrm{T}_{\mathrm{m} \text { ice }}=-9.0$ to $-10.0^{\circ} \mathrm{C}$ ) and homogenize into liquid phase in the temperature interval from 330 to $340^{\circ} \mathrm{C}$ (Fig. 9). Several inclusions with higher salinity $\left(\mathrm{T}_{\mathrm{m} \text { ice }}=-20.5\right.$ to $-21.0^{\circ} \mathrm{C}$; salinity $=22.8$ to $23.1 \mathrm{wt} . \% \mathrm{NaCl}$ equ.) have also been recorded (Fig. 9). Due todecrepitation before homogenization, usually between 320 and $340^{\circ} \mathrm{C}$, only a few total homogenizations into the liquid phase were obtained in the range from 360 to $380^{\circ} \mathrm{C}$ (Fig. 9). Secondary inclusions have $\mathrm{T}_{\mathrm{m} \text { hyd }}$ in the interval from -22.1 to $-26.0^{\circ} \mathrm{C}$ (the $\mathrm{NaCl}$ / $\left(\mathrm{NaCl}+\mathrm{CaCl}_{2}\right)$ mass ratio $\left.=52-89 \%\right), \mathrm{T}_{\mathrm{m} \text { ice }}$ between -2.0 and $-4.5^{\circ} \mathrm{C}$ (salinity $=3.4-7.1$ wt. $\% \mathrm{NaCl}$ equ.) and $\mathrm{T}_{\mathrm{H}}$ from 295 to $310^{\circ} \mathrm{C}$ (Fig. 9).

Quartz separated from the carbonate fragments within the main breccia (T1) was suitable for microthermometric investigations. Quartz is represented by euhedral crystals accompanied bydickite aggregates. Primary L-rich fluid inclusions homogenize to a liquid phase at temperature between 285 and $320^{\circ} \mathrm{C}$ (Fig. 9). Their salinities range from 6.0 to 8.5 wt.\% $\mathrm{NaCl}$ equ. (Fig. 9). Secondary inclusions have not been observed probably due to their small size. 
Table 3. Carbon and oxygen isotope composition of carbonates from the Trepča Pb-Zn-Ag skarn deposit.

\begin{tabular}{|c|c|c|c|c|c|}
\hline Sample & Level & Type of mineralization & Description & $\begin{array}{c}\delta^{13} \mathrm{C} \\
(\% 0, \mathrm{~V}- \\
\text { PDB) }\end{array}$ & $\begin{array}{c}\delta^{18} O \\
(\%, V- \\
\text { SMOW) }\end{array}$ \\
\hline \multicolumn{6}{|c|}{ Barren limestone } \\
\hline T6f & $\mathrm{X}$ & host limestone, barren, recrystallized & white recrystallized carbonate & 1.9 & 21.5 \\
\hline KM-10 & surface & host limestone, barren, recrystallized & greyish calcite crystals & 2.8 & 24.6 \\
\hline STS-0 & $\mathrm{X}$ & host limestone, barren, recrystallized & greyish recrystallized carbonate & 4 & 19.6 \\
\hline \multicolumn{6}{|c|}{ Carbonate from pyroclastic rocks } \\
\hline P1-1 & surface & caldera & matrix, grey calcite & -10.1 & 17.1 \\
\hline P1-2 & surface & caldera & white calcite grain & -3.7 & 15.6 \\
\hline P1-3 & surface & caldera & matrix & -3.9 & 16 \\
\hline $\mathrm{P} 2-1$ & surface & caldera & matrix & -7.2 & 16.6 \\
\hline $\mathrm{P} 2-2$ & surface & caldera & white calcite grain & 2.4 & 21 \\
\hline \multicolumn{6}{|c|}{ Travertine deposit } \\
\hline P4-1 & surface & travertine deposit & grayish to dark purple travertine & -1.1 & 19.6 \\
\hline P4-2 & surface & travertine deposit & grayish to dark purple travertine & -1.1 & 20 \\
\hline P4-3 & surface & travertine deposit & grayish to dark purple travertine & -1.2 & 20.1 \\
\hline P4-4 & surface & travertine deposit & grayish to dark purple travertine & -1.6 & 20.2 \\
\hline \multicolumn{6}{|c|}{ Carbonate from breccia } \\
\hline $\mathrm{T} 2-1$ & $\mathrm{X}$ & recrystallized limestone fragment & grey massive carbonate & -4.3 & 13.3 \\
\hline $\mathrm{T} 2-2$ & $\mathrm{X}$ & recrystallized limestone fragment & white massive carbonate & -4.4 & 12.6 \\
\hline T2a-1 & $\mathrm{X}$ & $\begin{array}{l}\text { recrystallized limestone fragment with } \\
\text { magnetite and arsenopyrite }\end{array}$ & white calcite grain (rhombohedral) & -4.5 & 13.5 \\
\hline \multicolumn{6}{|c|}{ Carbonate at contact with breccia } \\
\hline T3-1 & $\mathrm{X}$ & late phase calcite & white calcite crystals (elongated, scalenohedral) & 0.4 & 13.5 \\
\hline $\mathrm{T} 3-2$ & $\mathrm{X}$ & recrystallized limestone, barren & white massive carbonate & -2.8 & 12.5 \\
\hline $\mathrm{T} 7-4-1$ & $\mathrm{X}$ & recrystallized limestone, barren & greyish calcite crystals & 2.2 & 17.9 \\
\hline \multicolumn{6}{|c|}{ Hydrothermal parageneses, pre-ore } \\
\hline TR-VII-7 & VII & recrystallized carbonate & grey calcite crystals & 2.9 & 22.8 \\
\hline TR-XI-2-1 & $\mathrm{XI}$ & recrystallized carbonate & grey calcite crystals & 2.8 & 22.2 \\
\hline TR-XI-2-2 & $\mathrm{XI}$ & recrystallized carbonate & yellowish calcite crystals & -3.8 & 14.2 \\
\hline TR-XI-5-1 & $\mathrm{XI}$ & recrystallized carbonate & yellowish calcite crystals & 0.9 & 15.5 \\
\hline TR-XI-6-1 & $\mathrm{XI}$ & recrystallized carbonate & white calcite grain (rhombohedral) & -2.2 & 12.9 \\
\hline TR-XI-3-1 & $\mathrm{XI}$ & recrystallized carbonate & grey calcite crystals & 3 & 21 \\
\hline T9-5-A-1 & $\mathrm{X}$ & recrystallized carbonate & grey calcite grain & 2.3 & 20.9 \\
\hline \multicolumn{6}{|c|}{ Skarn parageneses, pre-ore } \\
\hline T6e & $\mathrm{X}$ & crystallized carbonate & white calcite crystals & -4.1 & 15.1 \\
\hline T9-6-1 & $\mathrm{X}$ & massive carbonate & yellow-pinkish massive carbonate & -3.9 & 12.9 \\
\hline \multicolumn{6}{|c|}{ Hydrothermal parageneses, syn-ore } \\
\hline $\begin{array}{l}\text { TR- } \\
\text { VIII-2-1 }\end{array}$ & VIII & carbonate impregnated with galena & white massive carbonate & -1.1 & 9.7 \\
\hline $\begin{array}{l}\text { TR- } \\
\text { VIII-4-1 }\end{array}$ & VIII & carbonate impregnated with galena & yellow massive carbonate & -3.8 & 15.5 \\
\hline TR-XI-4-2 & $\mathrm{XI}$ & carbonate co-existing with quartz & white calcite grain & 1.2 & 11.5 \\
\hline STS-1 & $\mathrm{X}$ & massive carbonate with mineralization & yellow-pinkish massive carbonate (light) & -3.7 & 15.4 \\
\hline STS-3 & $\mathrm{X}$ & massive carbonate & grey massive carbonate & -0.8 & 18.4 \\
\hline $\mathrm{T} 7-2-2$ & $\mathrm{X}$ & calcite cogenetic with sph mineralization & white calcite veins & 5.6 & 15 \\
\hline $148 \mathrm{~A}-1$ & $\mathrm{X}$ & calcite cogenetic with py mineralization & white calcite veins & 0.9 & 14 \\
\hline $\mathrm{T} 8-2-2$ & IX & calcite cogenetic with sph mineralization & white calcite veins & 1.3 & 10.8 \\
\hline T9-5-4 & $\mathrm{X}$ & carbonate impregnated with sph and py & white massive carbonate & -3.8 & 13.2 \\
\hline
\end{tabular}


Table 3. Continuated

\begin{tabular}{|c|c|c|c|c|c|}
\hline Sample & Level & Type of mineralization & Description & $\begin{array}{c}\delta^{13} C \\
(\% 0, V- \\
\text { PDB) }\end{array}$ & $\begin{array}{c}\delta^{18} O \\
(\% o, V- \\
\text { SMOW) }\end{array}$ \\
\hline \multicolumn{6}{|c|}{ Skarn parageneses, syn-ore } \\
\hline T6-W & $\mathrm{X}$ & $\begin{array}{l}\text { white carbonate associated with quartz } \\
\text { alteration of hedenbergite }\end{array}$ & white carbonate, fine-grained & 0.1 & 14.1 \\
\hline T6-Y & $\mathrm{X}$ & $\begin{array}{l}\text { yellow carbonate associated with quartz } \\
\text { alteration of hedenbergite }\end{array}$ & yellow carbonate, fine-grained & 1.3 & 15.8 \\
\hline T6-G & $\mathrm{X}$ & $\begin{array}{l}\text { grey carbonate associated with quartz } \\
\text { alteration of hedenbergite }\end{array}$ & grey carbonate, fine-grained & -2.5 & 11.8 \\
\hline T6a-1 & $\mathrm{X}$ & 1st carbonate after sph mineralization & pink & -4.6 & 14.4 \\
\hline T6a-2 & $\mathrm{X}$ & 2nd carbonate after sph mineralization & yellow massive carbonate & -0.1 & 11.2 \\
\hline T6a-3 & $\mathrm{X}$ & 3rd carbonate after sph mineralization & white massive carbonate & 1 & 14 \\
\hline T6b-1 & $\mathrm{X}$ & 1st carbonate after sph mineralization & pink & -4 & 14.1 \\
\hline T6b-2 & $\mathrm{X}$ & 2nd carbonate after sph mineralization & yellow massive carbonate & -0.2 & 9.9 \\
\hline T9-1-2 & $\mathrm{X}$ & carbonate in conntact with quartz & yellow massive carbonate & 0.9 & 9 \\
\hline T9-3-1 & $\mathrm{X}$ & carbonate in conntact with quartz & white massive carbonate & 1.3 & 8.9 \\
\hline T9-4-2 & $\mathrm{X}$ & $\begin{array}{l}\text { carbonate cogenetic with py mineraliza- } \\
\text { tion }\end{array}$ & yellow massive carbonate & -1.7 & 11.2 \\
\hline \multicolumn{6}{|c|}{ Hydrothermal parageneses, post-ore } \\
\hline TR-V-1 & $\mathrm{V}$ & last phase calcite & grey calcite crystals (elongated, scalenohedral) & 2.3 & 15.9 \\
\hline $\begin{array}{l}\text { TR- } \\
\text { VIII-2-2 }\end{array}$ & VIII & last phase calcite & white calcite crystals (elongated, scalenohedral) & 0.9 & 11.7 \\
\hline $\begin{array}{l}\text { TR- } \\
\text { VIII-4-2 }\end{array}$ & VIII & last phase calcite & white calcite crystals (elongated, scalenohedral) & 0.1 & 12.7 \\
\hline STS-2 & $\mathrm{X}$ & massive carbonate, late phase & yellow-pinkish massive carbonate (dark) & -3.8 & 15.4 \\
\hline T5-1 & $\mathrm{X}$ & last phase calcite & white calcite crystals (rhombohedral, vertical) & 1.8 & 15.5 \\
\hline $\mathrm{T} 7-2-1$ & $\mathrm{X}$ & last phase calcite & white calcite crystals (rhombohedral, vertical) & 3.2 & 14.6 \\
\hline $\mathrm{T} 7-2-3$ & $\mathrm{X}$ & last phase calcite & white calcite crystals (rhombohedral, vertical) & 2.3 & 14.5 \\
\hline T7-3a-1 & $\mathrm{X}$ & last phase calcite & white calcite crystals (rhombohedral) & 2.1 & 15.1 \\
\hline $\mathrm{T} 7-5-1$ & $\mathrm{X}$ & last phase calcite & white calcite crystals (rhombohedral) & 0.8 & 16.1 \\
\hline T8a-1 & IX & last phase calcite & white calcite crystals (rhombohedral) & 1.2 & 15.9 \\
\hline T8-2-1 & IX & last phase calcite & white calcite crystals (rhombohedral) & 0.9 & 16.3 \\
\hline T9-5-1 & $\mathrm{X}$ & last phase calcite ( $5 \mathrm{~mm}$ to $\mathrm{sph} \mathrm{min})$ & white calcite crystals (rhombohedral) & 0,5 & 10.2 \\
\hline T9-5-2 & $\mathrm{X}$ & last phase calcite ( $10 \mathrm{~mm}$ to $\mathrm{sph} \mathrm{min})$ & white calcite crystals (rhombohedral) & 1 & 13.9 \\
\hline T9-5-A-4 & $\mathrm{X}$ & last phase calcite & white calcite crystals (rhombohedral) & 1.3 & 15.7 \\
\hline T9-5-A-5 & $\mathrm{X}$ & phase before the last & white calcite crystals (rhombohedral) & -2.1 & 10.9 \\
\hline \multicolumn{6}{|c|}{ Skarn parageneses, post-ore } \\
\hline T6-1 & $\mathrm{X}$ & last phase calcite & white calcite crystals (rhombohedral) & 0.6 & 16.7 \\
\hline T9-1-1 & $\mathrm{X}$ & last phase calcite & white calcite crystals (rhombohedral, vertical) & -0.7 & 13.1 \\
\hline T9-4-1 & $\mathrm{X}$ & last phase calcite & white calcite crystals (rhombohedral, isometric) & 3 & 16.1 \\
\hline T9-4-3 & $\mathrm{X}$ & last phase calcite & white calcite crystals (rhombohedral, isometric) & -0.2 & 12.7 \\
\hline T9-6-2 & $\mathrm{X}$ & last phase calcite & white calcite crystals (rhombohedral) & -0.7 & 16 \\
\hline T9-6-(4)-1 & $\mathrm{X}$ & last phase calcite & white calcite crystals (rhombohedral, vertical) & -2.7 & 14.2 \\
\hline T9-6-(4)-2 & $\mathrm{X}$ & phase before the last & white calcite crystals (rhombohedral) & 1.6 & 9.1 \\
\hline
\end{tabular}

Two types of fluid inclusions were recognized in calcite grains hosted by pyroclastic material of the caldera (P1, P2): 1) Vapour-rich inclusions represent the majority of the inclusion population (Fig. 8g). This type of FIs are resistant to developing phases during extreme cooling to the temperature of liquid nitrogen and heating up to $400^{\circ} \mathrm{C}$, and 2) Two-phase aqueous fluid inclusions consist of vapour and liquid water at room temperature (Fig. 8h). Two-phase inclusions can be subdivided into liquid-rich and vapour-rich subtypes according to their vapour to liquid ratio. Coexistence of inclusions 
with miscellaneous degrees of filling suggests inhomogeneous trapping from boiling fluid (Fig. 7). The near-eutectic temperature observed within liquid-rich inclusions around $-50^{\circ} \mathrm{C}$ reflects the $\mathrm{CaCl}_{2}-\mathrm{NaCl}- \pm \mathrm{KCl}-\mathrm{H}_{2} \mathrm{O}$ compositional model. $\mathrm{T}_{\mathrm{m} \text { ice }}$ in the range between -4.2 and $-4.6^{\circ} \mathrm{C}$ corresponds to a salinity of between 6.7 and $7.3 \mathrm{wt} . \% \mathrm{NaCl}$ equ. (Fig. 9). Homogenizations recorded in the same temperature interval for liquid-rich and vapour-rich inclusions (320$340^{\circ} \mathrm{C}$; Fig. 9) suggest formation under boiling conditions.

\subsection{K/Ar DATA}

$\mathrm{K}$-Ar ages obtained on samples from the phreatomagmatic breccia at the Trepča $\mathrm{Pb}-\mathrm{Zn}-\mathrm{Ag}$ skarn deposit are reported in Table 4. Sanidine and whole rock analysis of hydrothermally altered quartz-latite gave two almost concordant ages of 24.0 \pm 0.7 and $25.1 \pm 0.9 \mathrm{Ma}$, respectively. Dating of the milled breccia reveals ages of $20.9 \pm 1.7$ and $23.2 \pm 0.7 \mathrm{Ma}$. The younger age is unreliable due to a low amount of radiogenic Ar and a large uncertainty.

\section{DISCUSSION}

This paper focuses on the breccia pipe and its role in ore-forming processes at the Trepča $\mathrm{Pb}-\mathrm{Zn}-\mathrm{Ag}$ skarn deposit. The P-T-X conditions during breccia emplacement were estimated from descriptive features, mineralogy, stable isotope and fluid inclusion data obtained from the breccia fragments and wall rocks.

The Trepča deposit was formed during three distinctive stages that are common for all types of skarn deposits elsewhere: (1) the stage of isochemical contact metamorphism during igneous rock emplacement, (2) the prograde stage characterized by metasomatic replacement of carbonate rocks with anhydrous silicate minerals, and (3) the retrograde hydrous stage that resulted in the alteration of earlier-formed mineral assemblages. Deposition of ore minerals accompanies stages 2 and 3 (e.g. MEINERT et al., 2005).

\subsection{THE STAGE OF ISOCHEMICAL CONTACT METAMORPHISM}

The isochemical contact metamorphism stage resulted in recrystallization of the country rocks (Upper Triassic limestone and schist, Fig. 2). The size of calcite grains in the recrystallized limestone varies from several millimetres, at places spatially distal to the mineralization and the breccia pipe, up to several centimetres near the contact with the ore bodies and the breccia. The absence of fluid inclusions from the barren recrystallization limestone suggests recrystallization without involvement of either $\mathrm{H}_{2} \mathrm{O}$ or $\mathrm{CO}_{2}$. The barren recrystallized limestone is characterized by a high $\mathrm{CaO}$ content and $\mathrm{REE}$ patterns with the negative $\mathrm{Ce}_{\mathrm{N}}$ anomaly that are typical for marine carbonates (ELDERFIELD \& GREAVES, 1982; HU et al., 1988). Furthermore, the average $\delta^{13} \mathrm{C}$ value of the barren recrystallized limestone corresponds well to the worldwide Phanerozoic marine carbonate values (VEIZER \& HOEFS, 1976) and $\delta^{18} \mathrm{O}$ values are consistent with the Triassic marine carbonate values (CLAYPOOL et al., 1980). Obviously, the chemical and isotope composition of the limestone were not significantly disturbed by the recrystallization processes.

\subsection{THE PROGRADE ANHYDROUS STAGE}

The host recrystallized limestone at the contact with the prograde (skarn) mineralization is depleted in ${ }^{13} \mathrm{C}$ and ${ }^{18} \mathrm{O}$, reflecting the contribution of magmatic $\mathrm{CO}_{2}$ (ZHENG \& HOEFS, 1993; ROSATELLI et al., 2010). The increased content of Fe, Mn, Mo, As, Au, Cs, Ga, REE and Y suggests that the listed elements were transported by infiltrating magmatic fluids.

\subsection{THE MAIN BRECCIA - LIMESTONE CONTACT}

Fluid inclusions are abundant in calcite crystals of the recrystallized limestone near contacts with the main breccia pipe and its lateral branches suggesting that aqueous fluids, channeled by the breccia, were involved in the recrystallization processes. Multiple generations of fluid inclusions that have been found at the contact with the main breccia pipe give an insight into the P-T-X characteristics of fluids involved in breccia formation processes. The high-temperature $\left(\mathrm{T}_{\mathrm{H}}=343\right.$ $370^{\circ} \mathrm{C}$ ) and high salinity (19.8-20.6 wt.\% $\mathrm{NaCl}$ equ.) L-rich fluid inclusion assemblages (Subtype 1a, Fig. 8a) represent the earliest among all recorded fluid inclusion generations. To keep fluids in the liquid phase at temperatures above $343^{\circ} \mathrm{C}$ the pressure should exceed $18 \mathrm{MPa}$ (Fig. 10). The high pressure possibly reflects lithostatic conditions that preceded breccia formation. The travertine deposits found at the Trepča deposit surface (approximately $850 \mathrm{~m}$ above mean sea level) mark the preserved palaeosurface and allow estimation of the palaeodepth. The analyzed recrystallized limestone-breccia contact (sample T3a, $75 \mathrm{~m}$ above mean sea level) occurs around $775 \mathrm{~m}$ below the palaeosurface implying the lithostatic pressure of $21 \mathrm{MPa}$. The pressure correction applied to the representative isochores (Fig. 10) suggests the formation temperature between 350 and $370^{\circ} \mathrm{C}$. The fluid inclusion assemblages that comprise coexisting L-rich and V-rich inclusions (Type 2, Fig. 8b) reflect boiling conditions probably related to decompression of the system from a lithostatic to hydrostatic regime due to the brecciation (STRMIĆ PALINKAŠ et al., 2014). Assuming the fluid salinity around 20 wt. $\% \mathrm{NaCl}$, the homogenization temperature between 285 and $305^{\circ} \mathrm{C}$ corresponds to adepth between 600 and 800 $\mathrm{m}$ below the former water table (Fig. 11). Boiling resulted in the separation of high salinity L-rich and low salinity V-rich inclusions. The fluid temperature drop from $350-370^{\circ} \mathrm{C}$ to $285-305^{\circ} \mathrm{C}$ suggests an adiabatic process and allows the calculation of the steam loss (e.g., SIMPSON et al., 2015):

$\mathrm{H}_{\mathrm{L}, \mathrm{T} f \text { (Subtype 1a) }}=(1-\mathrm{y}) \times \mathrm{H}_{\mathrm{L}, \text { Th(L-rich, Type 2) }}+\mathrm{y} \times \mathrm{H}_{\mathrm{V}, \mathrm{Th}(\mathrm{L} \text {-rich, Type 2) }}$

In this equation, $\mathrm{H}_{\mathrm{L}, \mathrm{Tf}(\text { Subtype 1a) }}$ represents an average specific enthalpy of the fluid before boiling at the temperature estimated from the pressure corrected representative isochores for Subtype 1a fluid inclusions (Fig. 10), $\mathrm{H}_{\mathrm{L}, \mathrm{Th}(\mathrm{L}-\mathrm{rich} \text {, }}$ Type 2) and $\mathrm{H}_{\mathrm{V}, \mathrm{Th}(\mathrm{L} \text {-rich, Type 2) }}$ are average specific enthalpies of liquid and vapor phases separated during boiling at temperature equal to the homogenization temperature of L-rich inclusions coexisting with V-rich inclusions, and $y$ is the steam (vapour) fraction. The specific enthalpies for liquid and vapour phases (assuming pure $\mathrm{H}_{2} \mathrm{O}$ ) were adopted from KEENAN et al. (1969). The calculated steam loss ranges around $30 \%$. The fluid inclusion assemblages composed 
Table 4. The K/Ar data obtained at the Trepča Pb-Zn-Ag skarn deposit.

\begin{tabular}{|l|l|l|l|l|l|l|}
\hline Sample & Sample description & Dated fraction & $\mathbf{K} \%$ & ${ }^{\mathbf{4 0}} \mathbf{A r} \mathbf{c m}^{\mathbf{3}} / \mathbf{g}$ & ${ }^{\mathbf{4 0}} \mathbf{A r} \mathbf{\%}$ & $\begin{array}{l}\text { K/Ar age } \\
\mathbf{( M a})\end{array}$ \\
\hline \multirow{2}{*}{ TR-6 } & $\begin{array}{l}\text { Surface, hydrothermaly } \\
\text { altered quartz-latite }\end{array}$ & Sanidine & 8.54 & $8.035 \times 10-6$ & 86.7 & $24.0 \pm 0.7$ \\
\cline { 2 - 7 } & Whole rock & 2.77 & $2.787 \times 10-6$ & 55.1 & $25.1 \pm 0.9$ \\
\hline T7-4a & Level X., milled breccia & Whole rock & 2.43 & $1.985 \times 10-6$ & 16.7 & $20.9 \pm 1.7$ \\
\hline T4a & Level X., milled breccia & Whole rock & 8.01 & $7.277 \times 10-6$ & 82.7 & $23.2 \pm 0.7$ \\
\hline
\end{tabular}

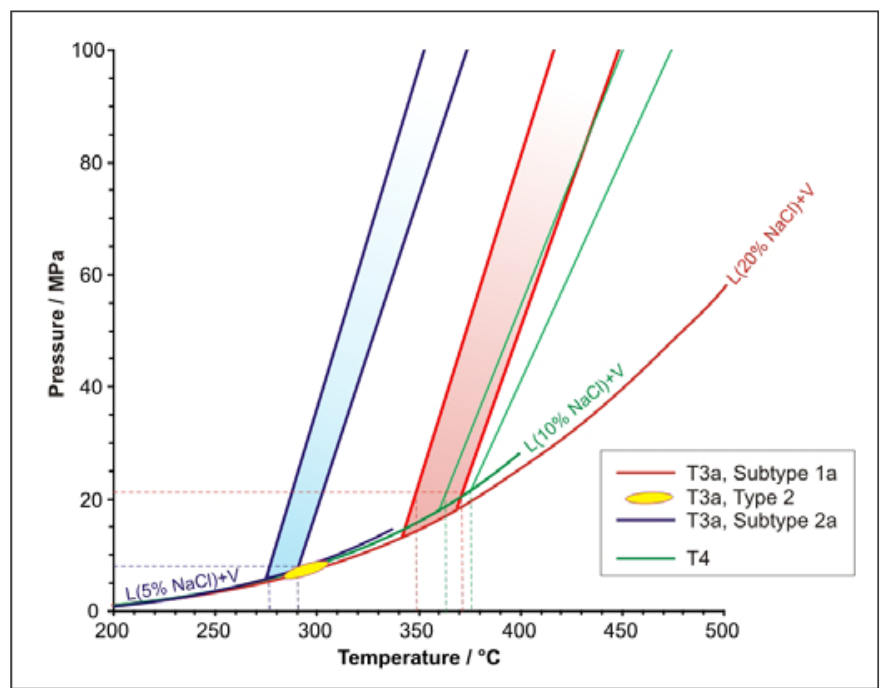

Figure 10. Pressure-temperature diagram showing the ranges of isochores for L-rich fluid inclusions hosted within the recrystallized limestone at its contact with the main breccia pipe (sample T3a, $75 \mathrm{~m}$ above mean sea level) and its contact with the lateral breccia branches (sample T4, 75 $\mathrm{m}$ above mean sea level). The boiling conditions obtained from the fluid inclusion assemblage composed of coexisting L-rich and V-rich inclusions are plotted too (Type 2, sample T3a, $75 \mathrm{~m}$ above mean sea level). The liquid-vapour curve for $\mathrm{H}_{2} \mathrm{O}-\mathrm{NaCl}$ fluids with 5, 10 and $20 \mathrm{wt} \% \mathrm{NaCl}$ is adopted from BODNAR \& VITYK (1994). only of V-rich inclusions (Type 3) reveal that fluids periodically were completely converted to vapour, probably due to episodic fluxes of hot magmatic fluids. Assuming the palaeodepth of around $775 \mathrm{~m}$, the pressure correction applied to the representative isochores for the moderate-temperature $\left(\mathrm{T}_{\mathrm{H}}=275-290^{\circ} \mathrm{C}\right)$ and low salinity (2.6-4.2 wt.\% $\mathrm{NaCl}$ equ.) L-rich fluid inclusion assemblages (Subtype 1b, Fig. X1) reveals their entrapment temperature between 275 and $290^{\circ} \mathrm{C}$. Cooling and dilution of the fluids may reflect a contribution of descending groundwaters in the later stages of breccia formation.

The recrystallized limestone at the contact with the main breccia pipe (T3a) shows an increase in $\mathrm{SiO}_{2}, \mathrm{Fe}_{2} \mathrm{O}_{3}$, and $\mathrm{MnO}$ content accompanied with the depletion in the $\mathrm{CaO}$ content comparing to the composition of the barren recrystallized limestone placed distally from the breccia. Some trace elements, including $\mathrm{Pb}, \mathrm{As}, \mathrm{Sb}, \mathrm{W}, \mathrm{Y}$ and REE are also enriched. The REE pattern possesses a weak negative $\mathrm{Ce}_{\mathrm{N}}$ anomaly and a prominent positive $\mathrm{Eu}_{\mathrm{N}}$ anomaly. Furthermore, the recrystallized limestone at the contact with the breccia is depleted in ${ }^{13} \mathrm{C}$ and ${ }^{18} \mathrm{O}$ indicating the contribution of isotopically light $\mathrm{CO}_{2}$ (Fig. 7) probably of a magmatic origin (ZHENG \& HOEFS, 1993; ROSATELLI et al., 2010).

\subsection{LATERAL BRECCIA BRANCHES - LIMESTONE CONTACT}

The lateral dyke-like branches infilled with the milled matrix represent non-venting branches of the main phreatomagmatic breccia (CORBETT \& LEACH, 1998). The hydraulic fracturing of country rocks requires a fluid pressure equal to the minimum principal stress plus the tensile strength of the rocks (JAEGER \& COOK, 1979). The Lrich fluid inclusions found in the recrystallized limestone at contacts with the fissures indicate that recrystallization processes occurred in the presence of moderate salinity single phase (liquid) fluids that were channeled through the fissures. The minimum entrapment pressure was around 20 MPa (Fig. 10) suggesting that the pressure was controlled by lithostatic conditions. The pressure correction applied to the representative isochores, assuming the lithostatic conditions (Fig. 10), suggests the entrapment temperature was between 365 and $375^{\circ} \mathrm{C}$. The abundant rock flour that was injected into such a type of fissures reflects transport by fluidization (McCALLUM, 1985; BRANQUET et al., 1999; CLARK \& JAMES, 2003). The various types of hydrothermal alterations, including sericitization, kaolinitization, pyritization and carbonatization, that affected the rock flour suggest an intensive hydrothermal circulation. The major element composition and the REE content of the recrystallized limestone at this type of contacts (samples T4 and T74, $75 \mathrm{~m}$ above mean sea level) overlap with the values obtained for the barren recrystallized limestone placed distally from the breccia. However, some trace elements, including $\mathrm{Pb}, \mathrm{Ni}, \mathrm{As}, \mathrm{Cd}$ and $\mathrm{Sb}$ are more abundant than in the barren limestone suggesting their transport by aqueous fluids. The $\delta^{13} \mathrm{C}$ values overlap with values recorded for the barren recrystallized limestone revealing the negligible contribution of magmatic $\mathrm{CO}_{2}$. In contrast, the decreased $\delta^{18} \mathrm{O}$ values may be attributed to the involvement of aqueous fluids. According to the fractionation equation for a calcite-water pair (ZHANG, 1999), the fluid in the isotopic equilibrium with the analyzed calcite $\left(\delta^{18} \mathrm{O}=17.9 \%\right)$ at temperatures around $370^{\circ} \mathrm{C}$ should have and ${ }^{18} \mathrm{O}$ value of $+13.7 \%$. 


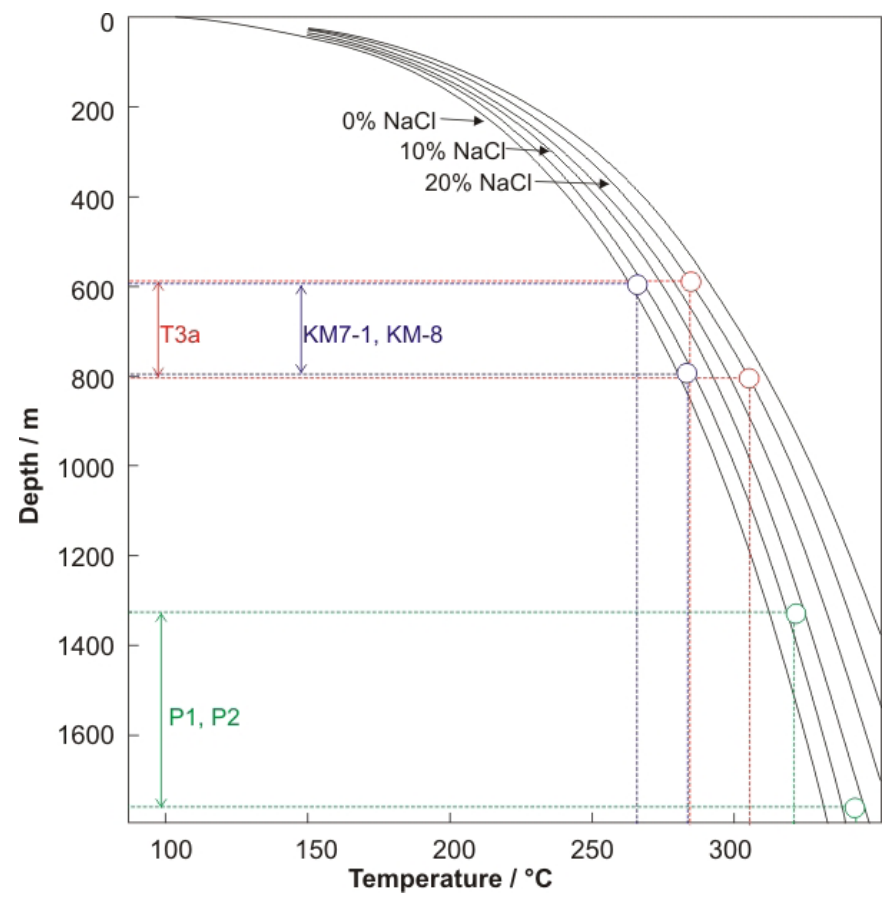

Figure 11. Fluid inclusion homogenization temperatures for coexistent liquid-rich and vapour-rich inclusions fitted on the boiling pointdepth curves for fluids of different salinities (after HAAS, 1971) estimate the formation depths assuming the hydrostatic regime.

\subsection{THE MAIN BRECCIA CLASTS}

The clasts of country rocks and skarn mineralization that preceded the breccia formation underwent intense milling, mixing and hydrothermal alterations depending on their lithological characteristics and spatial position within the breccia. They show mutual differences in shape, dimension, composition, alteration, direction and distance of transport typical for phreatomagmatic breccias (e.g. SILLITOE, 1985; BAKER et al., 1986; TAMAS \& MILESI, 2002). Sericitization of the breccia matrix, locally accompanied by minor kaolinitization, indicate increased water activity under near-neutral to weakly acidic conditions.

The fragments composed of a magnetite core and a pyrite rim (samples KM7-1, KM8; $835 \mathrm{~m}$ above mean sea level) suggest an increase in oxygen and sulfur fugacities over time (Fig. 12). Textural features suggest that the fibroradial magnetite core, accompanied by a mixture of quartz and carbonates, was formed by retrograde alteration of hedenbergite under increased oxygen fugacity:

$3 \mathrm{CaFe}^{2+} \mathrm{Si}_{2} \mathrm{O}_{6}+3 \mathrm{CO}_{2}+1 / 2 \mathrm{O}_{2} \rightarrow \mathrm{Fe}^{2+} \mathrm{Fe}^{3+}{ }_{2} \mathrm{O}_{4}+3 \mathrm{CaCO}_{3}+6 \mathrm{SiO}_{2}$ (2)

hedenbergite magnetite calcite quartz

The $f \mathrm{O}_{2}$ vs. $f \mathrm{~S}_{2}$ diagram constructed for the pre-breccia stage (lithostatic regime; $\mathrm{P} \approx 20 \mathrm{MPa} ; \mathrm{T} \approx 400^{\circ} \mathrm{C}$ ) and the main stage of the breccia formation (hydrostatic regime; $\mathrm{P} \approx 7.5 \mathrm{MPa} ; \mathrm{T} \approx$ $300^{\circ} \mathrm{C}$ ) reveals that hedenbergite stability strongly depends on temperature and pressure, favouring the prograde stage conditions (Fig. 12). The increased water activity and oxygen fugacity as well as the decreased temperature are direct consequences of the breccia pipe formation that enhanced the permeability along the recrystallized limestone-schist contact and enabled incursions of cold oxygenated groundwater in the system.

The presence of Bi-sulfides speaks in favour of the skarn precursor for these types of fragments. Namely, according to fluid inclusion data published by STRMIĆ PALINKAŠ et al. (2013), the prograde skarn mineralization was formed at temperatures between 390 and $475^{\circ} \mathrm{C}$. In contrast, the main ore deposition stage appears to have been largely contemporaneous with the retrograde stage of skarn development at temperatures below $350^{\circ} \mathrm{C}$. In weakly acidic to near-neutral aqueous solutions $\mathrm{Bi}$ is predominantly transported as the $\mathrm{Bi}(\mathrm{OH})_{3}{ }^{0}$ complex (SKIRROW \& WALSHE, 2002) and its mobility is negligible at temperatures below $400^{\circ} \mathrm{C}$ (TOOTH et al., 2013).

The pyrite rim reflects sulfidation of magnetite into pyrite under the increased sulfur fugacity:

$$
\begin{aligned}
& \mathrm{Fe}^{2+} \mathrm{Fe}_{2}^{3+} \mathrm{O}_{4}+3 \mathrm{~S}_{2}+8 \mathrm{H}^{+} \rightarrow 3 \mathrm{FeS}_{2}+4 \mathrm{H}_{2} \mathrm{O}(3) \\
& \text { magnetite } \\
& \text { pyrite }
\end{aligned}
$$

The isotope composition of sulfides from the Trepča $\mathrm{Pb}-\mathrm{Zn}$ Ag skarn deposit suggest a magmatic origin of sulfur (STRMIĆ PALINKAS̆ et al., 2013). The solubility of sulfur in silicate magma is a function of temperature, pressure, $\mathrm{FeO}$ and $\mathrm{SiO}_{2}$ content and oxygen fugacity (CARROLL \& WEBSTER, 1994; O'NEILL \& MAVROGENES, 2002; DE MOOR et al., 2013). Under reducing conditions, the sulfur solubility decreases with decreasing temperature, and cooling of magma plays an important role in sulfur degassing. Furthermore, at the Trepča Pb-ZnAg skarn deposit, phreatomagmatic explosions and formation of diatreme unsealed the system, decreased the pressure and additionally favouring sulfur release.

P-T-X characteristics of fluids involved in retrograde alteration processes are evidenced by multiple generations of fluid inclusions. Primary liquid-rich fluid inclusion assemblages in calcite grains entrap moderate salinity high temperature fluids. A significant increase in salinity recorded by the coexisting primary liquid- and vapour-rich inclusions may have been caused by boiling within an open system (e.g. SIMMONS \& BROWNE, 1997; BAUMGARTNER, 2008) and may not represent a fluid that originally had higher salinities. Alternatively, as reported by SIMMONS (1991) and ALBINSON et al. (2001) at the Fresnillo $\mathrm{Pb}-\mathrm{Zn}$-Ag deposit, Zacatecas, Mexico, the variation in salinity may have been caused by discrete pulses of saline fluids. The presence of numerous vapour-rich inclusions favours a boiling scenario that would also be consistent with the observed salinity variations without temperature change. Assuming hydrostatic conditions, boiling occurred at depths between 600 and $800 \mathrm{~m}$ (Fig. 11). The pseudosecondary liquid-rich fluid inclusion assemblages suggest dilution and cooling over time. Sporadic boiling episodes increased the salinity up to $0.3 \mathrm{wt} \% \mathrm{NaCl}$ (from 2.2-4.8 wt.\% $\mathrm{NaCl}$ equ. up to $2.6-5.1 \mathrm{wt} . \% \mathrm{NaCl}$ equ.) suggesting steam loss of between 6 and $15 \%$. The decreased $\delta^{13} \mathrm{C}$ and $\delta^{18} \mathrm{O}$ values obtained for calcite grains suggest an involvement of magmatic $\mathrm{CO}_{2}$ (Table 3, Fig. 7). Similar $\delta^{13} \mathrm{C}$ and $\delta^{18} \mathrm{O}$ values have been recorded for the recrystallized limestone fragments as well as for the recrystallized limestone that hosts the skarn mineralization (Table 3, Fig. 7).

The fragments composed of pyrite, pyrrhotite and minor Bisulfides reflect that locally increases in sulfur fugacity were sufficient to complete sulfidation of hedenbergite to pyrrhotite and/ or pyrite. Bismuth, a trace element in the skarn paragenesis, behaves in an immobile manner during the retrograde alteration processes accumulating in forms of various Bi-sulfides. 


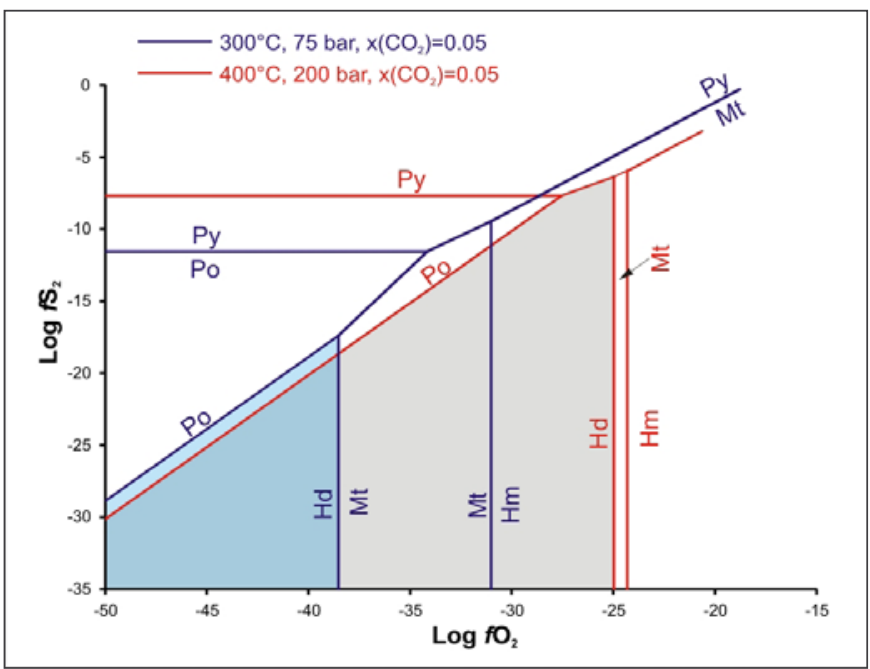

Figure 12. $\log f \mathrm{O}_{2}$ vs. $\log f \mathrm{~S}_{2}$ diagram of the Ca-Fe-Si-S-OH system constructed for the pre-breccia stage (lithostatic regime; $\mathrm{P} \approx 20 \mathrm{MPa}$; $\mathrm{T} \approx 400^{\circ} \mathrm{C}$ ) and the main stage of the breccia formation (hydrostatic regime; $\left.\mathrm{P} \approx 7.5 \mathrm{MPa} ; \mathrm{T} \approx 300^{\circ} \mathrm{C}\right)$. The $\mathrm{x}\left(\mathrm{CO}_{2}\right)$ value is set at 0.05 .

The limestone fragments found in the deeper portion of the breccia pipe are commonly replaced by siderite and occasionally impregnated by pyrite and microcrystalline quartz. Dickite aggregates and euhedral quartz occupy cavities. The fluid inclusion data obtained from quartz suggest a formation temperature of between 290 and $330^{\circ} \mathrm{C}$, from a fluid with salinity in the range of 6-8.5 wt \% $\mathrm{NaCl}$ equ. and a $\mathrm{pH}$ below 5.5 (STRMIĆ PALINKA $\breve{S}$ et al., 2009). The $\delta^{34} \mathrm{~S}$ value of pyrite suggests a magmatic source of sulfur (STRMIĆ PALINKAŠ, 2009).

Two distinctive generations of fluid inclusions in recrystallized limestone fragments accompanied with fibroradial arsenopyrite and magnetite suggest the dilution and cooling of hydrothermal fluids over time. The $\delta^{13} \mathrm{C}$ and $\delta^{18} \mathrm{O}$ values overlap with data obtained for calcite hosted by fragments composed of a magnetite core and pyrite rim, the recrystallized limestone fragments and the recrystallized limestone that hosts the skarn mineralization (Table 3, Fig. 7).

\subsection{HYDROTHERMAL CARBONATES}

The recrystallized limestone that hosts the hydrothermal mineralization shows a weak contribution of magmatic $\mathrm{CO}_{2}$ and slight enrichment in $\mathrm{Mn}$ and Fe. Concentration of other elements overlap with values obtained for the barren recrystallized Upper Triassic limestone.

Syn-ore and post-ore carbonates associated with both skarn and hydrothermal parageneses point to the diminishing influence of magmatic $\mathrm{CO}_{2}$ during the main-ore and post-ore stages (Table 3, Fig. 7). Syn-ore carbonates are enriched in $\mathrm{Fe}, \mathrm{Mg}$, $\mathrm{Mn}$, many chalcophile elements, including $\mathrm{Ag}, \mathrm{As}, \mathrm{Bi}, \mathrm{Cd}, \mathrm{Cu}$, $\mathrm{Pb}, \mathrm{Sb}$ and $\mathrm{Zn}$, as well as in $\mathrm{Au}, \mathrm{Y}$ and REE. Post-ore carbonates are depleted in $\mathrm{Fe}, \mathrm{Mg}, \mathrm{Mn}$ and the majority of chalcophile elements comparing to syn-ore carbonates, reflecting declining hydrothermal activity.

Traveritine, with traces of carbonates enriched in $\mathrm{Zn}$ and grains of galena, barite and gypsum, was precipitated during the late stage of hydrothermal activity. According to PENTECOST (2005), travertines are chemically-precipitated continental lime- stones where precipitation is mainly driven by $\mathrm{CO}_{2}$ degassing from a groundwater source leading to calcium carbonate supersaturation. Based on the origin of the $\mathrm{CO}_{2}$ interacting with the groundwater PENTECOST (2005) subdivided travertines into meteogene and thermogene deposits. Soil-zone and atmospheric $\mathrm{CO}_{2}$ may be characterized as meteoric in origin, since the terrestrial vegetation and associated soil contains carbon fixed from the atmosphere. Travertines formed from groundwaters charged with a meteoric carrier are termed meteogene whereas thermogene travertines are formed as a massive deposit from fluids carrying $\mathrm{CO}_{2}$ originating from thermal processes. Waters related to meteogene travertines have a lower temperature (generally ambient), a lower dissolved inorganic carbon content $(\mathrm{DIC}<10 \mathrm{mmol} / \mathrm{L})$, a lower $\mathrm{CO}_{2}$ partial pressure $\left(\mathrm{pCO}_{2}<0.01\right.$ $\mathrm{MPa}$ ) and $\mathrm{pH}$ values between 7 and 8 . Furthermore, while meteogene travertines generally display a negative carbon isotope composition $\left(\delta^{13} \mathrm{C}\right.$ between -12 and $0 \%$ ), thermogene travertines, in contrast, show $\delta^{13} \mathrm{C}$ values in the range of -1 to $10 \%$ (PENTECOST, 2005). On the basis of the geochemical characteristics the Trepča travertine should have been considered as thermogene in its origin although a contribution of organic-derived carbon cannot be excluded. Particularly, signs of rapid $\mathrm{CO}_{2}$ degassing during deposition such as bubbles and radial calcite crystals are typical ofthermogene travertine. The $\delta^{13} \mathrm{C}$ data mostly overlap with $\delta^{13} \mathrm{C}$ values previously published for thermogene travertines with a magmatic origin of $\mathrm{CO}_{2}$ (e.g. PENTECOST, 2005; D'ALESSANDRO et al., 2007). In addition, dissolution of the host recrystallized limestone during the main ore stage enriched hydrothermal fluids on $\mathrm{HCO}_{3}^{-}$. Furthermore, decreased $\delta^{13} \mathrm{C}$ values reflect the influence of organic carbon (e.g. LAZAREVA et al., 2008). The $\delta^{18} \mathrm{O}$ value is predominantly affected by a significant contribution of circulating groundwaters at elevated temperatures.

\subsection{PYROCLASTIC DEPOSITS}

According to their isotope composition, calcite grains hosted by pyroclastic material of the caldera represent minute fragments of the recrystallized Triassic limestone. In contrast, the carbonate matrix is significantly depleted in ${ }^{13} \mathrm{C}$ reflecting the contribution of organic carbon. Textural characteristics of pyroclastic material with fossil imprints of leaves and plant roots evidence precipitation within a lacustrine environment enriched in organic matter remains. However, a contribution of magmatic $\mathrm{CO}_{2}$ should not be ignored. Fluid inclusions hosted by calcite grains suggest their formation under boiling conditions. Homogenization temperatures between 320 and $340^{\circ} \mathrm{C}$ for fluid inclusions with salinities around $7 \mathrm{wt} . \% \mathrm{NaCl}$ equ. indicate the formation depth of approximately 500 - $650 \mathrm{~m}$ and 1300 - $1750 \mathrm{~m}$ assuming lithostatic and hydrostatic conditions (Fig. 11), respectively. Younger V-rich inclusions reflect a sudden pressure drop probably related to phreatomagmatic explosions.

\subsection{THE K/Ar AGE}

The presence of the hydrothermally altered quartz-latite dyke within the main breccia core and overlapping K/Ar ages of hydrothermally altered quartz-latite $(24.0 \pm 0.7 \mathrm{Ma}$ and $25.1 \pm 0.9$ $\mathrm{Ma})$ and the milled matix breccia $(23.2 \pm 0.7 \mathrm{Ma})$ suggest that magmatic activity coincided with the mineralizing events. The volcanic rocks in the area, produced by the Late Oligocene post- 
collisional magmatic activity (CVETKOVIĆ et al., 2004), display mostly overlapping $\mathrm{K} / \mathrm{Ar}$ (this study) and $\mathrm{Ar} / \mathrm{Ar}$ ages (BOROJEVIĆ ŠOŠTARIĆ et al., 2012).

\section{CONCLUSIONS}

According to the mineralogical, geochemical and isotope data we can distinguish several stages that directed formation of the phreatomagmatic breccia pipe at the Trepča $\mathrm{Pb}-\mathrm{Zn}-\mathrm{Ag}$ skarn deposit:

1) The stage of isochemical contact metamorphism resulted from emplacement of a magmatic chamber during the Late Oligocene postcollisional magmatic activity. This stage preceded the mineralization and the breccia formation and resulted in the recrystallization of country rocks without significant changes in their chemical and isotope composition.

2) The stage of prograde (skarn) mineralization preceded the breccia formation. The prograde (skarn) mineralization resulted from the interaction of magmatic fluids with the recrystallized Upper Triassic limestone. The magmatic fluids were derived from a cooling magmatic body below the ore deposit. The host recrystallized limestone at the contact with the prograde (skarn) mineralization has an increased $\mathrm{Fe}, \mathrm{Mn}, \mathrm{Mo}$, As, $\mathrm{Au}$, $\mathrm{Cs}, \mathrm{Ga}, \mathrm{REE}$ and $\mathrm{Y}$ content suggesting their transport by infiltrating magmatic fluids. The decreased $\delta^{13} \mathrm{C}$ and $\delta^{18} \mathrm{O}$ values reflect the contribution of magmatic $\mathrm{CO}_{2}$. In addition, the pyroxene predominant mineralogy reflects a low oxygen fugacity and a low water activity.

3) Emplacement of the quartz-latite dyke along the contact between the recrystallized limestone and the overlying schist. An interaction of the hot magmatic body with cold ground water resulted in phreatomagmatic explosions and formation of the phreatomagmatic breccia. The pyroclastic deposits found at the surface of the Trepča $\mathrm{Pb}-\mathrm{Zn}$ - Ag skarn deposit confirm subaqueous explosive volcanic activity.

The contact between the main breccia pipe and the host recrystallized limestone has preserved geochemical and isotope evidence of variable P-T-X conditions during the phreatomagmatic brecciation. The early generations of fluid inclusions precede the brecciation and reflect the recrystallization under lithostatic conditions at temperatures between 350 and $370^{\circ} \mathrm{C}$. The phreatomagmatic brecciation turned the system from the lithostatic to hydrostatic regime, decreasing the pressure and triggering the fluid boiling. Assuming an adiabatic process, the temperature drop from $350-370^{\circ} \mathrm{C}$ to $285-305^{\circ} \mathrm{C}$ resulted with a steam loss of approximately $30 \%$. The later generations of fluid inclusions point to cooling and dilution of the fluids due to an enhanced contribution of descending groundwaters in late stages of the breccia formation. The host recrystallized limestone at the contact with the main breccia pipe is enriched in $\mathrm{Si}$, $\mathrm{Fe}, \mathrm{Mn}, \mathrm{Pb}, \mathrm{As}, \mathrm{Sb}, \mathrm{W}, \mathrm{REE}$ and $\mathrm{Y}$ and depleted in ${ }^{13} \mathrm{C}$ and ${ }^{18} \mathrm{O}$.

The lateral dyke-like branches infilled with the milled matrix represent non-venting branches of the main phreatomagmatic breccia. The fluid inclusions founded in the recrystallized limestone at contacts with the fissures indicate that hot $\left(365-375^{\circ} \mathrm{C}\right)$ and moderate salinity single phase aqueous (liquid) fluids enriched in $\mathrm{Pb}, \mathrm{Ni}, \mathrm{As}, \mathrm{Cd}$ and $\mathrm{Sb}$ were channeled through the fissures under lithostatic conditions.
4) The hydrothermally altered quartz-latite dyke points to an increase in water activity over time. Partly preserved sanidine accompanied with the mixture of muscovite and quartz reflects a near-neutral to weakly acidic environment.

5) The clasts of country rocks and skarn mineralization underwent intense milling and mixing due to repeated magmatic penetrations. Sericitization of the breccia matrix, locally accompanied with minor kaolinitization, point to an increased water activity under near-neutral to weakly acidic conditions. Large fragments originally composed of anhydrous skarn minerals (pyroxenes) are usually completely altered to a mixture of fibroradial magnetite, quartz and various amount of carbonates suggesting an increase in oxygen fugacity. Their pyrite rims reflect that the increase in the oxygen fugacity was followed by an increase in sulfur fugacity. The fragments predominantly composed of Fe-sulfides and minor Bi-sulfides indicate that locally increases in sulfur fugacity were sufficient to complete sulfidation of hedenbergite to pyrrhotite and/or pyrite. The increase in water activity and oxygen fugacity are associated with the breccia pipe formation that enhanced the permeability along the recrystallized limestone-schist contact and enabled groundwater incursions in the system. At the same time, cooling and decompression of the system intensified the magmatic sulfur degassing.

7) Syn-ore and post-ore carbonates associated with both skarn and hydrothermal parageneses reflect the diminishing influence of magmatic $\mathrm{CO}_{2}$ during the main-ore and post-ore stages. Syn-ore carbonates are enriched in $\mathrm{Fe}, \mathrm{Mg}, \mathrm{Mn}$, many chalcophile elements, including $\mathrm{Ag}, \mathrm{As}, \mathrm{Bi}, \mathrm{Cd}, \mathrm{Cu}, \mathrm{Pb}, \mathrm{Sb}$ and $\mathrm{Zn}$, as well as in $\mathrm{Au}, \mathrm{Y}$ and REE. In contrast, post-ore carbonates reflect a declining hydrothermal activity and the depletion in $\mathrm{Fe}, \mathrm{Mg}, \mathrm{Mn}$ and the majority of chalcophile elements.

8) Travertine deposits were precipitated in the late stage of hydrothermal activity at the Trepča Pb-Zn-Ag skarn deposit. Their isotope signature suggests that carbon originates predominantly from the dissolute host recrystallized limestone influenced by significant amounts of magmatic and organic carbon. In contrast, $\delta^{18} \mathrm{O}$ values are strongly affected by the contribution of circulating groundwaters at elevated temperatures.

Although the phreatomagmatic breccia at the Trepča $\mathrm{Pb}-$ $\mathrm{Zn}$-Ag skarn deposit does not carry significant amounts of the ore mineralization, its formation was crucial for ore deposition. Phreatomagmatic explosions and formation of the breccia triggered the retrograde stage increasing the water activity and oxygen fugacity in the system. In addition, cooling and decompression of the system contributed to more effective degassing of magmatic sulfur increasing the sulfur fugacity.

\section{Acknowledgement}

This study was supported by the Croatian Ministry of Sciences, Technology and Sports (Projects 119-0982709-1175). We are grateful to the geological teams of the Trepča deposit, in particular to M. DIEHL for help and constructive discussions during fieldwork. We would especially like to thank reviewers, Vasilios MELFOS and Irina MARINOVA, whose comments improved the paper. 


\section{REFERENCES}

ALBINSON, T., NORMAN, D.I., COLE, D. \& CHOMIAK, B. (2001): Controls on formation of low-sulfidation epithermal deposits in Mexico: Constraints from fluid inclusion and stable isotope data.- Society of Economic Geologist Special Publication, 8, 1-32.

ANDERSON, E.D., ATKINSON JR, W.W., MARSH, T. \& IRIONDO, A. (2009): Geology and geochemistry of the Mammoth breccia pipe, Copper Creek mining district, southeastern Arizona: evidence for a magmatic-hydrothermal origin.- Miner. Deposita, 44/2, 151-170. doi: 10.1007/ s00126-008-0206-2

BAKER, E.M., KIRWIN, D.J. \& TAYLOR, R.G. (1986): Hydrothermal breccia pipes.- EGRU Contribution 12, James Cook University of North Queensland, Australia, 45p.

BAKER, E.M. \& ANDREW, A.S. (1991): Geologic, fluid inclusion and stable isotope studies of the gold-bearing breccia pipe at Kidston, Queensland, Australia.- Econ. Geol., 86/4, 810-830. doi: 10.2113/gsecongeo.86.4.810

BALOGH, K. (1985): K/Ar dating of Neogene volcanic activity in Hungary: experimental technique, experiences and methods of chronological studies.-ATOMKI report, Debrecen, D/1, 277-288.

BAUMGARTNER, R., FONTBOTÉ, L. \& VENNEMANN, T. (2008): Mineral zoning and geochemistry of epithermal polymetallic $\mathrm{Zn}-\mathrm{Pb}-\mathrm{Ag}-\mathrm{Cu}-\mathrm{Bi}$ mineralization at Cerro de Pasco, Peru.- Econ. Geol., 103/3, 493-537. doi: 10.2113/gsecongeo.103.3.493

BODNAR, R.J. (2003): Introduction to fluid inclusions.- Min Assoc Can Short Course Series, 32, 1-8.

BODNAR, R.J. \& VITYK, M.O. (1994): Interpretation of microthermometric data for $\mathrm{H}_{2} \mathrm{O}-\mathrm{NaCl}$ fluid inclusions.- In: DE VIVO, B. \& FREZZOTTI, M.L. (eds.): Fluid Inclusions in Minerals: Methods and Applications. Short Course of the Working Group (IMA) Inclusions in Minerals, Virginia Polytechnic Institute and State University, Blacksburg, Virginia, $117-130$.

BODNAR, R.J., REYNOLDS, T.J. \& KUEHN, C.A. (1985): Fluid inclusion systematics in epithermal systems.- Rev. Econ. Geol., 2, 73-98.

BORISENKO, A.S. (1977): Study of the salt composition of solutions of gasliquid inclusions in minerals by the cryometric method.- Geol.Geofiz., 18, 16-27.

BOROJEVIĆ ŠOŠTARIĆ, S., CVETKOVIĆ, V., NEUBAUER, F., PALINKAS̆, L.A., BERNROIDER, M. \& GENSER, J. (2012) Oligocene shoshonitic rocks of the RogoznaMts.(Central Balkan Peninsula): Evidence of petrogenetic links to the formation of $\mathrm{Pb}-\mathrm{Zn}-\mathrm{Ag}$ ore deposits.Lithos, 148/1, 176-195.

BOROJEVIĆ ŠOŠTARIĆ, S., PALINKAŠ, L.A., NEUBAUER, F., HURAI, V., CVETKOVIĆ, V., ROLLER-LUTZ, Z., MANDIĆ, M. \& GENSER, J. (2013): Silver-base metal epithermal vein and listwanite hosted deposit Crnac, Rogozna Mts., Kosovo, part II: A link between magmatic rocks and epithermal mineralization.- Ore Geol. Rev., 50/1, 98-117.

BOROJEVIĆ ŠOŠTARIĆ, S., PALINKAS̆, A.L., NEUBAUER, F., CVETKOVIĆ, V., BERNROIDER, M. \& GENSER, J. (2014): The origin and age of the metamorphic sole from the Rogozna Mts., Western Vardar Belt: New evidence for the one-ocean model for the Balkan ophiolites.Lithos, 192/1, 39-55.

BRANQUET, Y., CHEILLETZ, A., GIULIANI, G., LAUMONIER, B. \& BLANCO, O. (1999): Fluidized hydrothermal breccia in dilatant faults during thrusting: the Colombian emerald deposits.- Geological Society, London, Special Publications, 155, 183-195. doi: 10.1144/GSL. SP.1999.155.01.14

BYRNE, K. \& TOSDAL, R.M. (2014): Genesis of the Late Triassic southwest zone breccia-hosted alkalic porphyry $\mathrm{Cu}$-Au deposit, Galore Creek, British Columbia, Canada.- Econ. Geol., 109/4, 915-938. doi: 10.2113/econgeo.109.4.915

CANET, C., GONZÁLEZ-PARTIDA, E., CAMPRUBÍ, A., CASTRO-MORA, J., ROMERO, F.M., PROL-LEDESMA, R.M., LINARES, C., ROMERO-GUADARRAMA, J.A. \& SÁNCHEZ-VARGAS, L.I. (2011): The $\mathrm{Zn}-\mathrm{Pb}-\mathrm{Ag}$ skarns of Zacatepec, Northeastern Oaxaca, Mexico: A study of mineral assemblages and ore-forming fluids.- Ore Geol. Rev., 39/4, 277-290.

CANNELL, J., COOKE, D.R., WALSHE, J.L. \& STEIN, H. (2005): Geology, mineralization, alteration, and structural evolution of the E1 Teniente porphyry Cu-Mo deposit.- Econ. Geol., 100/5, 979-1003. doi: 10.2113/gsecongeo.100.5.979

CARROLL, M.R. \& WEBSTER, J.D. (1994): Solubilities of sulfur, noble gases, nitrogen, chlorine, and fluorine in magmas.- Rev. Miner. Geochem., 30, 231-279.

CLARK, C. \& JAMES, P. (2003): Hydrothermal brecciation due to fluid pressure fluctuations: examples from the Olary Domain, South Australia.Tectonophysics, 366/3, 187-206. doi: 10.1016/S0040-1951(03)00095-7

CLAYPOOL, G.E., HOLSER, W.T., KAPLAN, I.R., SAKAI, H. \& ZAK, I. (1980): The age curves of sulfur and oxygen isotopes in marine sulfate, and their mutual interpretation.- Chem Geol, 28/1, 199-259. doi: 10.1016/0009-2541(80)90047-9
COOKE, D.R. \& BLOOM, M.S. (1990): Epithermal and subjacent porphyry mineralization, Acupan, Baguio district, Philippines: a fluid-inclusion and paragenetic study.- J. Geochem.Explor., 35/1, 297-340. doi: 10.1016/0375-6742(90)90042-9

CORBETT, G.J. \& LEACH, T.M. (1998): Southwest Pacific rim gold-copper systems: Structure, alteration, and mineralization.- Society of Economic Geologists Special Publication, 6, 234.

CVETKOVIĆ, V., KARAMATA, S. \& KNEŽEVIĆ, V. (1995): Volcanic rocks of the Kopaonik District.- Geology and Metallogeny of the Kopaonik Mt. Symposium, Belgrade, 185-194.

CVETKOVIĆ, V., PRELEVIĆ, D., DOWNES, H., JOVANOVIĆ, M., VASELLI, O. \& PECSKAY, Z. (2004): Origin and geodynamic significance of Tertiary postcollisional basaltic magmatism in Serbia (central Balkan Peninsula).- Lithos, 73/3-4, 161-186. doi: 10.1016/j.lithos.2003.12.004

D'ALESSANDRO, W., GIAMMANCO, S., BELLOMO, S. \& PARELLO, F. (2007): Geochemistry and mineralogy of travertine deposits of the SW flank of Mt. Etna (Italy): Relationships with past volcanic and degassing activity.- J. Volcanol. Geoth. Res., 165/1-2, 64-70. doi: 10.1016/j.jvolgeores.2007.04.011

DAVIES, A.G., COOKE, D.R., GEMMELL, J.B. \& SIMPSON, K.A. (2008): Diatreme breccias at the Kelian gold mine, Kalimantan, Indonesia: Precursors to epithermal gold mineralization.- Econ. Geol., 103/4, 689-716. doi: 10.2113/gsecongeo.103.4.689

DE MOOR, J.M., FISCHER, T.P., SHARP, Z.D., KING, P.L., WILKE, M. BOTCHARNIKOV, R.E., COTTRELL, E., ZELENSKI, M., MARTY, B. KLIMM, K., RIVARD, C., AYALEW, D., RAMIREZ, C. \& KELLEY, K.A. (2013): Sulfur degassing at Erta Ale (Ethiopia) and Masaya (Nicaragua) volcanoes: Implications for degassing processes and oxygen fugacities of basaltic systems.- Geochem. Geophy. Geosy., 14/10, 4076-4108. doi: 10.1002 /ggge. 20255

DILEK, Y., FURNES, H. \& SHALLO, M. (2007): Suprasubduction zone ophiolite formation along the periphery of Mesozoic Gondwana.- Gondwana Res., 11/4, 453-475. doi: 10.1016/j.gr.2007.01.005

DIMITRIJEVIĆ, M.D. (2001): The Dinarides and the Vardar zone: A short review of the geology.- Acta Vulcanol., 13/1, 1-8.

ELDERFIELD, H. \& GREAVES, M.J. (1982): The rare earth elements in seawater- Nature 296, 214-219. doi: 10.1038/296214a0

FÉRAUD, J., MALIQI, G. \& MEHA, V. (2007): Famous mineral localities: the Trepca mine, Stari Trg, Kosovo.- Mineral. Rec., 38/4, 267-298.

GOLDSTEIN, R.H. (2001): Fluid inclusions in sedimentary and diagenetic systems.- Lithos, 55/1, 159-193. doi: 10.1016/S0024-4937(00)00044-X

GOLDSTEIN, R.H. \& REYNOLDS, T.J. (1994): Systematics of fluid inclusions in diagenetic minerals.- Society for Sedimentary Geology, Short Course, 31,199 p. doi: $10.2110 /$ scn. 94.31

HAAS, J.L. (1971): The effect of salinity on the maximum thermal gradient of a hydrothermal system at hydrostatic pressure.- Econ. Geol., 66/6, 940946. doi: 10.2113/gsecongeo.66.6.940

HENLEY, R.W. (1984): Chemical structure of geothermal systems.- Rev. Econ. Geol., 1, 73-98.

HU, X., WANG, Y.L. \& SCHMITT, R.A. (1988): Geochemistry of sediments on the Rio Grande Rise and the redox evolution of the south Atlantic Ocean.- Geochim. Cosmochim. Acta, 52, 201-207. doi: 10.1016/00167037(88)90068-3

JAEGER, J.C. \& COOK, N.G.W. (1979): Fundamentals of rock mechanics.Chapman and Hall, London, $593 \mathrm{p}$.

JANKOVIĆ, S. (1997): The Carpatho-Balkanides and adjacent area: A sector of the Tethyan Eurasian metallogenic belt.- Miner. Deposita, 32/5, 426-433.

KARAMATA, S., MAJER, V. \& PAMIĆ, J. (1980): Ophiolites of Yugoslavia.- Ofioliti, 1/1, 105-125.

KARAMATA, S., OLUJIĆ, J., PROTIĆ, LJ., MILOVANOVIĆ, D. VUJNOVIĆ, L., POPEVIĆ, A., MEMOVIĆ, E., RADOVANOVIĆ, Z. \& RESIMIĆ-ŠARIĆ, K.(2000): The Western belt of the Vardar zone the remnant of a marginal sea.- In: KARAMATA, S. \& JANKOVIĆ, S. (eds.): Geology and metallogeny of the Dinarides and the Vardar zone: Zvornik, Academy of Science and Arts of Republika Srpska, 131-135.

KEENAN, J.H., KEYES, F.G., HILL, P.G. \& MOORE, J.G. (1969): Steam Tables.- John Wiley, New York, 1962 p.

KOŁODZIEJCZYK, J., PRŠEK, J., MELFOS, V., VOUDOURIS, P.C., MALIQI, F. \& KOZUB-BUDZYŃ, G. (2015): Bismuth minerals from the StanTerg deposit (Trepça, Kosovo).- Neues Jb. Miner. Abh., 192/3, $317-333$.

LANDTWING, M.R., DILLENBECK, E.D., LEAKE, M.H. \& HEINRICH, C.A. (2002): Evolution of the breccia-hosted porphyry Cu-Mo-Au deposit at Agua Rica, Argentina: Progressive unroofing of a magmatic hydrothermal system.- Econ. Geol., 97/6, 1273-1292. doi: 10.2113/gsecongeo.97.6.1273

LAZAREVA, E.V., BRYANSKAYA, A.V., ZHMODIK, S.M., PONOMARCHUK, V.A., BARKHUTOVA, D.D. \& SEMIONOVA, D.V. (2008): Carbon isotope composition in microbial mats and travertine of BRZ.- Geochim.Cosmochim.Acta, 72/12, A521. 
LORENZ, V. (1973): On the formation of maars.- Bull.Volcanol., 37/2, 183-204. doi: 10.1007/BF02597130

MALO, M., MORITZ, R., DUBE, B., CHAGNON, A., ROY, F. \& PELCHAT, C. (2000): Base metal skarns and Au occurrences in the southern Gaspé Appalachians: distal products of a faulted and displaced magmatic-hydrothermal system along the Grand Pabos-Restigouche fault system.- Econ. Geol., 95/6, 1297-1318.

McCALLUM, M.E. (1985): Experimental evidence for fluidization processes in breccia pipe formation.- Econ. Geol., 80/6, 1523-1543. doi: 10.2113/ gsecongeo.80.6.1523

MEINERT, L.D. (1992): Skarns and skarn deposits.- Geosci. Can., 19/4, $145-162$

MEINERT, L.D., DIPPLE, G.M. \& NICOLESCU, S. (2005): World skarn deposits.- Econ. Geol. 100 ${ }^{\text {th }}$ Anniversary Volume, 299-336.

MILOVANOVIĆ, D., MARCHIG, V. \& KARAMATA, S. (1995): Petrology of the crossite schist from Fruška Gora Mts. (Yugoslavia), relic of a subducted slab of the Tethyan oceanic crust.- J. Geodyn., 20/3, 289-304.

NANCE, W.B. \& TAYLOR, S.R. (1976): Rare earth element patterns and crustal evolution: I. Australian post-Archean rocks.- Geochim. Cosmochim. Acta, 40/12, 1539-1551. doi: 10.1016/0016-7037(76)90093-4

PENTECOST, A. (2005): Travertine.- Springer-Verlag, Berlin Heidelberg, $445 \mathrm{p}$.

ROBERTSON, A.H., TRIVIĆ, B., ĐERIĆ, N. \& BUCUR, I.I. (2013): Tectonic development of the Vardar ocean and its margins: Evidence from the Republic of Macedonia and Greek Macedonia.- Tectonophysics, 595/1, 25-54. doi: 10.1016/j.tecto.2012.07.022

ROSATELLI, G., WALL, F., STOPPA, F. \& BRILLI, M. (2010): Geochemical distinctions between igneous carbonate, calcite cements, and limestone xenoliths (Polino carbonatite, Italy): spatially resolved LAICPMS analyses.- Contrib. Mineral. Petr., 160/5, 645-661. doi: 10.1007/s00410-010 0499-x

SAMSON, I.M. \& WALKER, R.T. (2000): Cryogenic Raman spectroscopic studies in the system $\mathrm{NaCl}-\mathrm{CaCl}_{2}-\mathrm{H}_{2} \mathrm{O}$ and implications for low-temperature phase behaviour in aqueous fluid inclusions.- Can. Mineral., 38/1, $35-43$.

SCHUMACHER, F. (1950): Die Lagerstätte der Trepča und ihre Umgebung.Izdavačko Preduzeće Saveta za Energetiku i Ekstraktivnu Industriju vlade FNRJ, Beograd, $65 \mathrm{p}$.

SCHUMACHER, F. (1954): The ore deposits of Yugoslavia and the development of its mining industry.- Econ. Geol., 49/5, 451-492.

SHARP, I.R. \& ROBERTSON, A.H. (2006): Tectonic-sedimentary evolution of the western margin of the Mesozoic Vardar Ocean: evidence from the Pelagonian and Almopias zones, northern Greece.- Geological Society, London, Special Publications, 260/1, 373-412. doi: 10.1144/GSL. SP.2006.260.01.16

SILLITOE, R.H. (1985): Ore-related breccias in volcanoplutonic arcs.- Econ. Geol., 80/6, 1467-1514. doi: 10.2113/gsecongeo.80.6.1467

SILLITOE, R.H., BAKER, E.M. \& BROOK, W.A. (1984): Gold deposits an hydrothermal eruption breccias associated with a maar volcano at Wau, Papua New Guinea.- Econ. Geol., 79/4, 638-655. doi: 10.2113/gsecongeo.79.4.638

SIMMONS, S.F. (1991): Hydrologic implications of alteration and fluid inclusion studies in the Fresnillo district, Mexico: Evidence for a brine reservoir and a descending water table during the formation of hydrothermal Ag-Pb-Zn orebodies.- Econ. Geol., 86/8, 1579-1601. doi: 10.2113/gsecongeo.86.8.1579

SIMMONS, S.F. \& BROWNE, P.R.L. (1997): Saline fluid inclusions in sphalerite from the Broadlands-Ohaaki geothermal system: A coincidental trapping of fluids being boiled toward dryness.- Econ. Geol., 92/4, 485-489. doi: 10.2113 /gsecongeo.92.4.485

SIMPSON M.P., STRMIĆ PALINKAŠ S., MAUK J.L., BODNAR R.J. (2015): Fluid inclusion chemistry of adularia-sericite epithermal $\mathrm{Au}-\mathrm{Ag}$ deposits of the southern Hauraki Goldfield, New Zealand.- Econ. Geol., 110/3, 763-786.

SKEWES, M.A., HOLMGREN, C. \& STERN, C.R. (2003): The Donoso copper-rich, tourmaline-bearing breccia pipe in central Chile: petrologic fluid inclusion and stable isotope evidence for an origin from magmatic fluids.- Miner. Deposita, 38/1, 2-21. doi: 10.1007/s00126-002-0264-9

SKIRROW, R.G. \& WALSHE, J.L. (2002): Reduced and oxidized Au-Cu-Bi iron oxide deposits of the Tennant Creek Inlier, Australia: An integrated geologic and chemical model.- Econ. Geol., 97/6, 1167-1202. doi: 10.2113/gsecongeo.97.6.1167

STERNER, S.M., HALL, D.L. \& BODNAR, R.J. (1988): Synthetic fluid inclusions. V. Solubility relations in the system NaCl-KCl- $\mathrm{H}_{2} \mathrm{O}$ under vapor-saturated conditions.- Geochim. Cosmochim. Acta, 52/5, 989-1005. doi: 10.1016/0016-7037(88)90254-2

STRMIĆ PALINKAŠ, S. (2009): Genesis of hydrothermal and skarn paragenesis in the Pb-Zn-Ag mineral deposit Trepča (StariTrg), Kosovo.- Unpublished $\mathrm{PhD}$ thesis, University of Zagreb, Zagreb, $143 \mathrm{p}$.
STRMIĆ PALINKAS̆, S., PALINKAS̆, L.A., MOLNAR, F. \& DIEHL, M. (2007): Basic research in service of successful exploitation in $\mathrm{Pb}-\mathrm{Zn}-\mathrm{Ag}$ Stari Trg mine, Trepča, Kosovo.- In: ANDREW, C.J. (ed.): Digging deeper: Navan, Ireland, Irish Association for Economic Geology, 165-167.

STRMIĆ PALINKAŠ, S., BOROJEVIĆ ŠOŠTARIĆ, S., BERMANEC, V., PALINKAŠ, L., PROCHASKA, W., FURIĆ, K. \& SMAJLOVIĆ, J. (2009): Dickite and kaolinite in the $\mathrm{Pb}-\mathrm{Zn}-\mathrm{Ag}$ sulphide deposits of northern Kosovo (Trepča and Crnac).- Clay Miner., 44/1, 67-79.

STRMIĆ PALINKAS̆, S., PALINKAŠ, L.A., RENAC, C., SPANGENBERG, J.E., LÜDERS, V., MOLNAR, F. \& MALIQI, G. (2013): Metallogenic model of the Trepča $\mathrm{Pb}-\mathrm{Zn}-\mathrm{Ag}$ skarn deposit, Kosovo: evidence from fluid inclusions, rare earth elements, and stable isotope data.- Econ. Geol., 108/1, 135-162.

STRMIĆ PALINKAŠ, S., WEGNER, R., ČOBIĆ, A., PALINKAŠ, L.A., DE BRITO BARRETO, S., VÁCZI, T. \& BERMANEC, V. (2014): The role of magmatic and hydrothermal processes in the evolution of Be-bearing pegmatites: Evidence from beryl and its breakdown products.- Am. Mineral., 99/2-3, 424-432.

SUDAR, M. (1986):Mikrofosili I biostratigrafija trijasaunutrašnjih Dinarida između Gučeva I Ljubišnje (Triassic microfossils and biostratigraphy of the Inner Dinarides between Gučevo and Ljubišnja Mts., Yugoslavia; in Serbian with English summary).- Geol. An. Balk.Poluos., 50, 151-394.

SUDAR, M. \& KOVACS, S. (2006): Metamorphosed and ductilely deformed conodonts from Triassic limestones situated beneath ophiolite complexes: Kopaonik Mountain (Serbia) and Bükk Mountains (NE Hungary)-a preliminary comparison.- Geol.Carpath., 57/3, 157-176.

TAMAS, C.G. \& MILESI, J.-P. (2002): Hydrovolcanic breccia pipe structures-General features and genetic criteria. I. Phreatomagmatic breccias.Studia UBB Geologia, 47/1, 127-147.

TERZIĆ, S.B., SOMMERAUER, J. \&HARNIK, A.B. (1974): Macroscopic cosalite crystals from the $\mathrm{Pb}-\mathrm{Zn}$ ore deposit Trepča (Yugoslavia).- Schweiz. Mineral. Petrogr. Mitt., 54/1, 209-211.

TOOTH, B., ETSCHMANN, B., POKROVSKI, G.S., TESTEMALE, D., HAZEMANN, J.-L., GRUNDLER, P.V. \& BRUGGER, J. (2013): Bismuth speciation in hydrothermal fluids: An X-ray absorption spectroscopy and solubility study.- Geochim. Cosmochim. Acta, 101/1, 156-172. doi: 10.1016/j.gca.2012.10.020

TORNOS, F., CASQUET, C., GALINDO, C., VELASCO, F. \& CANALES, A. (2001): A new style of Ni-Cu mineralization related to magmatic breccia pipes in a transpressional magmatic arc, Aguablanca, Spain.- Miner. Deposita, 36/7, 700-706. doi: 10.1007/s001260100204

TRUMBULL, R.B., SLACK, J.F., KRIENITZ, M.S., BELKIN, H.E. \& WIEDENBECK, M. (2011): Fluid sources and metallogenesis in the Blackbird $\mathrm{Co}-\mathrm{Cu}-\mathrm{Au}-\mathrm{Bi}-\mathrm{Y}-\mathrm{REE}$ district, Idaho, USA: insights from major-element and boron isotopic compositions of tourmaline.- Can. Mineral., 49/1, 225-244.

VASKOVIĆ, N. \& MATOVIĆ, V. (2010): Ophiolites of the Vardar Zone and the Dinarides: Central and West Serbia.- IMA 2010 field trip guide RS1. Department of Mineralogy, Geochemistry and Petrology, University of Szeged, $55 \mathrm{p}$.

VEIZER, J.D. \& HOEFS, J. (1976): The nature of ${ }^{18} \mathrm{O} /{ }^{16} \mathrm{O}$ and ${ }^{13} \mathrm{C} /{ }^{12} \mathrm{C}$ secular trends in sedimentary carbonate rocks.- Geochim. Cosmochim. Acta, 40/11, 1387-1395.

VESELINOVIC-WILLIAMS, M. (2011): Characteristics and origin of polymetallic mineralisation in the Kopaonik region of Serbia and Kosovo, with particular reference to the Belo Brdo Pb-Zn (Ag) deposit.- Unpublished $\mathrm{PhD}$ thesis, Kingston University, London, $150 \mathrm{p}$.

VRY, V.H., WILKINSON, J.J., SEGUEL, J. \& MILLÁN, J. (2010): Multistage intrusion, brecciation, and veining at El Teniente, Chile: Evolution of a nested porphyry system.- Econ. Geol., 105/1, 119-153. doi: 10.2113/ gsecongeo.105.1.119

WALLIER S, REY R, KOUZMANOV K, PETTKE T, HEINRICH CA, LEARY S, O'CONNOR G, TĂMAS CG, VENNEMANN T, ULLRICH $\mathrm{T}$ (2006): Magmatic fluids in the breccia-hosted epithermal Au-Ag deposit of Roşia Montană, Romania.- Econ. Geol., 101/5, 923-954.

YANG, K.\& BODNAR, R.J. (1994): Why is economic porphyry copper mineralization absent from the granitoids of the Gyeongsang Basin, South Korea? Evidence from silicate melt and aqueous fluid inclusions.- Int. Geol. Rev., 36/7, 608-628.

ZELIĆ, M., AGOSTINI, S., MARRONI, M., PANDOLFI, L. \& TONARINI, S. (2010): Geological and geochemical features of the Kopaonik intrusive complex (Vardar zone, Serbia).- Ofioliti, 35/1, 33-47.

ZHENG, Y.F. (1999): Oxygen isotope fractionation in carbonate and sulfate minerals.- Geochem. J., 33, 109-126. doi: 10.2343/geochemj.33.109

ZHENG, Y.F. \& HOEFS, J. (1993): Carbon and oxygen isotopic covariations in hydrothermal calcites.- Miner. Deposita, 28/2, 79-89. doi: 10.1007/ BF00196332 